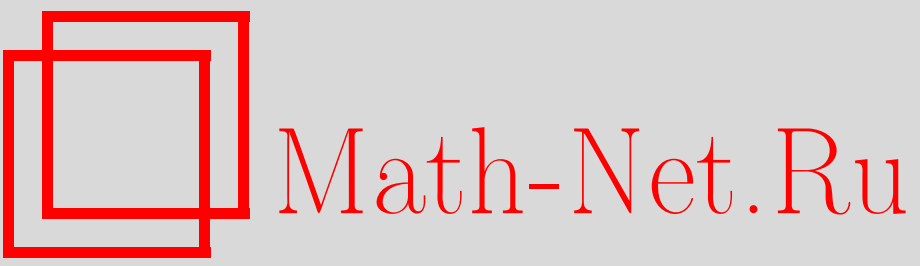

И. В. Пылюк, Критическое поведение трехмерной изинговской системы. Зависимость термодинамических характеристик от микроскопических параметров, TMФ, 1998, том 117, номер 3, 442-470

DOI: https://doi.org/10.4213/tmf945

Использование Общероссийского математического портала Math-Net.Ru подразумевает, что вы прочитали и согласны с пользовательским соглашением

http://www.mathnet.ru/rus/agreement

Параметры загрузки:

IP : 54.198.67.100

26 апреля 2023 г., 09:55:32 
ТЕОРЕТИЧЕСКАЯ

И МАТЕМАТИЧЕСКАЯ

ФИЗИКА

Том 117, № 3

декабрь, 1998

(C) 1998 г.

И. В. Пылюк*

\section{КРИТИЧЕСКОЕ ПОВЕДЕНИЕ ТРЕХМЕРНОЙ ИЗИНГОВСКОЙ СИСТЕМЫ. ЗАВИСИМОСТЬ ТЕРМОДИНАМИЧЕСКИХ ХАРАКТЕРИСТИК ОТ МИКРОСКОПИЧЕСКИХ ПАРАМЕТРОВ}

Представлены результаты исследования критического поведения трехмерной модели Изинга методом коллективных переменных.

\section{1. ВВЕДЕНИЕ}

Проблема описания фазовых переходов и критических явлений и построение микроскопической теории фазовых переходов являются одними из центральных задач статистической физики. В результате интенсивных исследований фазовых переходов в последнее время были сформулированы новые концепции, связанные с пониманием сути критических явлений, создан математический аппарат их описания. Вышеуказанным вопросам посвяшен целый ряд монографий [1-15], успехи в их решении суммированы также в обзорных статьях [16-18] и недавно появившихся работах и книгах [19-22].

В данной статье дан анализ некоторых новых работ, посвященных развитию теории фазовых переходов и критических явлений. Основное внимание обрашено на исследование критического поведения трехмерной модели Изинга. Приведены основные результаты одного из способов описания критических свойств этой модели.

Наиболее ярким достижением теории критических явлений, которое подтверждает концепию универсальности и скейлинга, является развитие идей ренормализационной группы (РГ) [5]. Использование Вильсоном техники фейнмановских диаграмм, а также введение нового параметра разложения $\epsilon=4-d$ и конструктивной идеи о том, что критические показатели плавно меняются с изменением размерности пространства $d$, открыли путь для развития в теории критических явлений методов теоретико-полевой РГ, которая подробно обсуждается в книге [23]. Применение РГ-подхода в теории критических явлений привело к более глубокому пониманию процессов, происходящих в окрестности критической температуры, дало возможность рассчитывать критические показатели, уравнения состояния, корреляционные функции и другие важные характеристики разнообразных модельных систем. В настоящее время сушествуют разные

* Институт физики конденсированных систем Национальной академии наук Украины, Львов, Украина 
формулировки РГ-рассмотрения критических явлений. Суть некоторых подходов изложена в книгах $[6,8,10,15,24]$. Сушественных успехов теория критических явлений достигла при использовании перенормированных рядов теории возмущений [25] и применении процедуры пересуммирования к асимптотическим рядам $[26,27]$.

Обширность литературы по теории фазовых переходов не позволяет детально остановиться на всех существующих методах их исследования. Весомое значение имеет появление монографии [28], в которой впервые собраны и систематизированы результаты, относящиеся к решеточным моделям, допускающим точное аналитическое решение. Несомненный интерес представляют работы, связанные с использованием разложений по степеням $1 / d[29], 1 / n[8,30]$, а также метода функционального интегрирования [31], с помошью которого без применения теории возмушений для $d$-мерной системы с вырожденным $n$-компонентным параметром порядка построено приближенное дифференциальное уравнение РГ [32], линеаризация которого в окрестности неподвижной точки произведена в работе [33]. Успешно используются методы РГ в реальном пространстве [34, 35], вариационные подходы $[36,37]$, метод функций Грина $[38,39]$. Наряду с исследованием критических явлений в ферро- и антиферромагнетиках [40-42] большое внимание уделяется фазовым превращениям в бинарных растворах $[43,44]$, гамильтониан решеточной модели которых представляет собой обобщение модели Изинга [45]. Описание феноменологических моделей фазовых переходов можно осуществлять методами теории катастроф [46]. Использованию понятий скейлинга и универсальности при описании статистических систем посвящен обзор [47]. Ряд методов расчета термодинамических функций для класса решеточных моделей, включающего в себя и модель Изинга, обсуждается в [48]. В [49] на основе разложения выражения для свободной энергии по спиновым кластерам систематически излагается приближение рядов по этим кластерам, которые сродни приближению среднего поля. В рамках этого приближения вычислены критическая температура и критические показатели для изинговских систем разной размерности. При этом использован метод когерентной аномалии. Применимость метода когерентной аномалии к разложениям в ряды проверяется в [50] с помощью пробных функций и расчета критического показателя восприимчивости для модели Изинга. Обзор литературы, касающейся проверки условий применимости приближения среднего поля при анализе критических явлений, дан в статье [51]. В работе [52] приведен обзор классификации фазовых переходов и ее связей с основами статистической физики.

Свое дальнейшее развитие при изучении фазовых переходов получают численные методы. Для трехмерной модели Изинга со взаимодействием ближайших соседей вьполнено детальное численное исследование универсальных отношений амплитуд восприимчивости, корреляционной длины и теплоемкости [53]. Ведущие поправки к скейлинговым амплитудам этих величин в случае разных типов решеток обсуждаются в [54]. Новый численный метод для определения природы фазового перехода предложен в [55], а в [56] описан новый численный подход к исследованию критических свойств моделей Изинга размерностью больше двух, основанный на расчете матрицы перехода. Среди численных методов изучения решеточных моделей в статистической механике активно используется метод Монте-Карло. Так, исследованию модели Изинга на простой кубической решетке методом РГ в процедуре Монте-Карло посвящена статья [57]. В работе [58] приводятся результаты анализа методом Монте-Карло корреляционной длины 
трехмерной модели Изинга для ОЦК-решетки и ренормализационного взаимодействия в критической области. Метод локальных состояний для вычисления свободной энергии решеточных моделей при использовании метода Монте-Карло представлен в [59]. Расчеты с помошью приведенного здесь нового метода дают надежные результаты даже в критических областях и не требуют большой вычислительной работы. В основе метода лежат использование верхней и нижней гранищ термодинамического предела плотности энтропии, в результате чего получается надежная оценка величины свободной энергии, а также выражение для величины допускаемой ошибки. Действенность метода проверяется на двух- и трехмерных моделях Изинга и на трехмерной модели Поттса с тремя состояниями. В статье [60] модель Изинга на простой кубической решетке изучается с помошью алгоритма кластерного обновления Монте-Карло. Улучшение результатов вычислений достигнуто в процессе исследования критического поведения трехмерной модели Изинга методом РГ в процедуре Монте-Карло [61] и высокоточным методом Монте-Карло [62, 63]. В рамках последнего в [64] изучаются некоторые универсальные отношения амплитуд для случая трехмерной модели Изинга. Наряду с методом Монте-Карло важное место при исследовании критических явлений в последнее время продолжает уделяться низкотемпературным и высокотемпературным разложениям. При них расчеты осушествляются в виде разложений интересуюших нас функций либо по возрастаюшим степеням температуры (низкотемпературное разложение), либо по степеням обратной температуры (высокотемпературное разложение). В [65] при рассмотрении поведения изинговского ферромагнетика с бесконечным спином получено низкотемпературное разложение для свободной энергии, формулы для теплоемкости, спонтанной намагниченности и магнитной восприимчивости в нулевом поле. В этой же статье из высокотемпературного разложения свободной энергии найдено выражение для высокотемпературной магнитной восприимчивости. В качестве примера можно привести и работу [66], где расчет критического показателя теплоемкости трехмерной модели Изинга осушествляется с использованием высокотемпературного разложения с точностью до 24-го порядка. Результаты, полученные методами высокотемпературного и низкотемпературного разложений, представляются в настоящее время наиболее достоверными, хотя нет строгого доказательства сходимости возникающих здесь рядов или оценки остатка ряда. Анализ этих рядов можно проводить с помощью техники аппроксимант Паде (см., например, [4]). Методы разложения в ряд дают возможность получить численные предсказания для трехмерных решеточных моделей, не поддаюшихся точному решению, оценить пригодность микроскопической модели путем сравнения полученных для нее результатов с опытом.

В большинстве работ, посвященных вопросам теории фазовых переходов, основное внимание уделялось определению класса универсальности систем, исследованию симметрийных свойств безотносительно к затравочным параметрам исходного гамильтониана, типов решений рекуррентных соотношений (РC) и вычислению значений критических показателей. Получены важные экспериментальные результаты. Рассчитаны универсальные отношения и комбинации критических амплитуд термодинамических характеристик спиновых систем, в частности трехмерной модели Изинга. Проблема зависимости самих же критических амплитуд от микроскопических параметров системы требовала последовательного изучения и могла быть успешно разрешена вместе с реше- 
нием основного вопроса теории фазовых переходов - получением явных выражений термодинамических характеристик системы вблизи точки фазового перехода как функций температуры и микроскопических параметров. Сушественный прогресс в решении этой проблемы был достигнут в методе коллективных переменных (КП), обобщенном Юхновским $[11,67-69]$ на случай спиновых систем. Первоначально этот метод теоретического исследования системы заряженных частиц был изложен в работах Бома и Пайнса (см., например, [70]), а также Зубарева [71]. Впоследствии он использовался при изучении бозе- и ферми-систем [72, 73], при построении статистической теории классических равновесных систем [74], при исследовании жидких магнетиков [75] и квазирелятивистских систем [76]. При описании металлических систем предложен метод динамических КП [77]. Метод КП успешно применяется при построении статистической теории такой неупорядоченной системы, как бинарные сплавы замешения [78], теории сегнетоэлектрического фазового перехода в кластерных системах [79], для исследования критического поведения системы жидкость-газ [80], бинарных смесей [81], $n$-компонентной модели классического магнетика $[82,83]$. Развитию РГ-преобразования в рамках метода КП и анализу этого преобразования с помощью теории возмущений посвящена работа [84]. Метод КП оказался плодотворным при изучении критических свойств трехмерной модели Изинга и при развитии на примере этой модели одного из способов расчета и анализа на микроскопическом уровне явных выражений для термодинамических и структурных функций однокомпонентной спиновой системы в реальном трехмерном пространстве. Остановимся на исследовании критического поведения трехмерной модели Изинга в методе КП. Опишем основную идею и схему расчета термодинамических характеристик трехмерной изинговской системы вблизи критической температуры $T_{\mathrm{c}}$ и исследование их зависимости от микроскопических параметров системы. Далее с учетом этой и предыдущих работ укажем на полученные основные результаты.

КП - это собирательное название особого класса переменных, специфических для каждой отдельной физической системы $[11,85]$. Множество КП содержит переменные, связанные с параметрами порядка. Благодаря этому фазовое пространство КП является наиболее естественным для описания фазового перехода. Для магнитных систем КП $\rho_{\mathbf{k}}$ - это переменные, связанные с модами колебаний плотности спинового момента, а с параметром порядка связана переменная $\rho_{0}$, индекс нуль которой отвечает максимуму фурье-образа потенциала взаимодействия.

В рассматриваемом методе КП исходным пунктом постановки задачи служит гамильтониан трехмерной модели Изинга. Далее осуществляется переход к множеству КП, вычисление якобиана перехода от спиновых переменных к КП приводит к функционалу статистической суммы, сходному с функционалом Гинзбурга-Ландау. РГ-преобразование, выполняемое в данном подходе, можно отнести к вильсоновскому типу. Основной особенностью является интегрирование коротковолновых мод, которое ведется в общем случае без применения теории возмушений.

Метод КП позволяет провести приближенный расчет выражения для статистической суммы трехмерной модели Изинга, получить, кроме универсальных величин (критических показателей), полные выражения для термодинамических функций в окрестности $T_{\mathrm{c}}$.

Основная идея расчета явных выражений для свободной энергии и других термоди- 
намических функций системы состоит в раздельном учете вкладов от коротковолновых и длинноволновых мод колебаний плотности спинового момента $[11,86,87]$. Коротковолновые моды характеризуются наличием РГ-симметрии и описываются негауссовой плотностью меры. Последняя представляется в виде экспонентной функции от КП $\rho_{\mathbf{k}}$, в аргументе которой наряду с квадратичным слагаемым присутствуют высшие степени переменной с соответствуюшими постоянными взаимодействия. Простейшей негауссовой мерой является четверная (модель $\rho^{4}$ ), содержашая в показателе экспоненты вторую и четвертую степени переменной. За ней следует шестерная (модель $\rho^{6}$ ), в которой имеется дополнительное слагаемое, пропорциональное шестой степени переменной, и т.д. Коротковолновым модам соответствует область критического режима (КР), имеющая место как вьше, так и ниже $T_{c}$. Здесь используется метод РГ $[5,8]$. За счет учета коротковолновых мод колебаний плотности спинового момента происходит перенормировка дисперсии распределения, описываюшего длинноволновые моды. Послед-

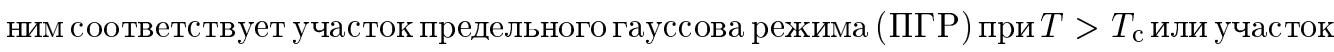
инверсного гауссова режима (ИГР) при $T<T_{c}$. В области ПГР флуктуации характеризуются гауссовым распределением. ИГР в отличие от ПГР описывается негауссовой плотностью меры. Только в результате выделения свободной энергии упорядочения распределение принимает гауссов характер. Способ учета вклада длинноволновых мод колебаний в свободную энергию системы качественно отличается от методики вычисления коротковолновой части статистической суммы. Расчет этого вклада основан на использовании гауссовой плотности меры в качестве базисной.

Ниже схема вычисления свободной энергии трехмерной изинговской системы дана в приближении шестерной плотности меры. При этом обсуждается целесообразность использования указанного приближения.

\section{2. СХЕМА РАСЧЕТА СВОБОДНОЙ ЭНЕРГИИ СИСТЕМЫ}

Рассматривается трехмерная модель Изинга на простой кубической решетке с периодом $c$. Гамильтониан модели имеет вид

$$
H=-\frac{1}{2} \sum_{\mathbf{j}, \mathbf{l}} \Phi(|\mathbf{j}-\mathbf{l}|) \sigma_{\mathbf{j}} \sigma_{\mathbf{l}},
$$

где $\Phi(|\mathbf{j}-\mathbf{l}|)$ - потенциал взаимодействия частиц, находящихся в узлах $\mathbf{j}$ и $\mathbf{l}, \sigma_{\mathbf{j}}-$ оператор $z$-компоненты спина в $\mathbf{j}$-м узле, имеюший два собственных значения +1 и -1 . Потенциал взаимодействия представляется экспоненциально убывающей функцией

$$
\Phi\left(r_{\mathbf{j} \mathbf{1}}\right)=A \exp \left(-\frac{r_{\mathbf{j} \mathbf{l}}}{b}\right) .
$$

Здесь $A$ - постоянная, $r_{\mathbf{j} \mathbf{1}}$ - расстояние между частицами, $b$ - радиус эффективного взаимодействия. Для фурье-образа потенциала взаимодействия используется аппроксимация

$$
\widetilde{\Phi}(k)= \begin{cases}\widetilde{\Phi}(0)\left(1-2 b^{2} k^{2}\right), & k \leqslant B^{\prime}, \\ 0, & B^{\prime}<k \leqslant B,\end{cases}
$$

где $B$ - гранища полузоны Бриллюэна $(B=\pi / c), B^{\prime}=(b \sqrt{2})^{-1}, \widetilde{\Phi}(0)=8 \pi A(b / c)^{3}$. 
В представлении КП для статистической суммы трехмерной модели Изинга имеem $[11]$

$$
Z=\int \exp \left[\frac{1}{2} \sum_{\mathbf{k}} \beta \widetilde{\Phi}(k) \rho_{\mathbf{k}} \rho_{-\mathbf{k}}\right] J(\rho)(d \rho)^{N} .
$$

Здесь суммирование по волновым векторам $\mathbf{k}$ производится в пределах первой зоны Бриллюэна, $\beta=1 /(k T)$ - обратная температура, КП $\rho_{\mathbf{k}}$ вводятся с помощью соотношений типа аналитического функционала для операторов мод колебаний спиновой плотности

$$
\hat{\rho}_{\mathbf{k}}=(\sqrt{N})^{-1} \sum_{\mathbf{l}} \sigma_{\mathbf{l}} \exp (-i \mathbf{k} \mathbf{l})
$$

$$
\begin{aligned}
J(\rho)= & 2^{N} \int \exp \left[2 \pi i \sum_{\mathbf{k}} \omega_{\mathbf{k}} \rho_{\mathbf{k}}+\sum_{n=1}^{\infty}(2 \pi i)^{2 n} N^{1-n} \times\right. \\
& \left.\times \frac{\mathcal{M}_{2 n}}{(2 n) !} \sum_{\mathbf{k}_{1}, \ldots, \mathbf{k}_{2 n}} \omega_{\mathbf{k}_{1}} \ldots \omega_{\mathbf{k}_{2 n}} \delta_{\mathbf{k}_{1}+\cdots+\mathbf{k}_{2 n}}\right](d \omega)^{N}
\end{aligned}
$$

- якобиан перехода от множества $N$ спиновых переменных $\sigma_{\mathbf{l}}$ к множеству КП $\rho_{\mathbf{k}}$, $\delta_{\mathbf{k}_{1}+\cdots+\mathbf{k}_{2 n}}-$ символ Кронекера. Переменные $\omega_{\mathbf{k}}$ сопряжены с $\rho_{\mathbf{k}}$, а кумулянты $\mathcal{M}_{2 n}$ принимают постоянные значения [11]. Выражение для статистической суммы (2.4) из-за присутствия в экспоненте (2.5) бесконечного числа слагаемых точно рассчитать нельзя. Поэтому используются приближения, связанные с ограничением числа слагаемых в экспоненте подынтегрального выражения (2.5). Определенная аппроксимация подынтегральной функции в выражении для $J(\rho)$ при вычислении явного вида якобиана перехода определяет выбор модели (модель $\rho^{4}, \rho^{6}$ и т.д.). При $n=1$ получаем гауссово приближение. Оно приводит к классическим значениям критических показателей. Важным условием описания критических свойств модели Изинга является использование негауссовых плотностей мер. Простейшее приближение, позволяюшее выйти за рамки классического поведения, соответствует $n=2$ и основывается на использовании четверной плотности меры (модель $\rho^{4}$ ). Для него осуществлен расчет основных критических показателей термодинамических характеристик и полных выражений для этих характеристик с учетом конфлюэнтных поправок, а также проанализированы соотношения для критических амплитуд [87-89]. Из-за приближенности расчета статистической суммы в связи с ограничением моделью $\rho^{4}$ полученные результаты (критические показатели, амплитуды, термодинамические функции) содержат определенную зависимость от параметра РГ $s$. Данная зависимость существенно уменьшается в процессе усложнения формы негауссовой плотности меры. Последнее подтверждается расчетом критического показателя коррелящионной длины $\nu$ для моделей $\rho^{2 m}(m=3,4,5)$ [90-94], а также непосредственным сравнением графиков температурных зависимостей термодинамических функций, рассчитанных для моделей $\rho^{4}$ и $\rho^{6}$ при разных значениях параметpa $s$ [95]. Результаты проведенных исследований показывают, что в интервале промежуточных значений $s(2 \leqslant s \leqslant 4)$ качественную картину критического поведения дает модель $\rho^{4}$. Следуюшая по сложности модель $\rho^{6}(n=3$, см. (2.5)), предусматриваюшая 
при интегрировании статистической суммы учет шестерной плотности меры, позволяет осушествить количественное описание критических свойств спиновой системы. Отметим, что разностная форма РС, основанная на негауссовой плотности меры, хорошо работает именно для промежуточных значений параметра РГ $s$. Для малых значений $s(s \rightarrow 1)$ в разложениях для функций, входяших в РС, сушественно иметь правильный единичный элемент. Для его выделения РС следует представить в виде рядов теории возмушений относительно гауссова распределения. При $s^{-3} \ll 1$ “проблема" единичного элемента не возникает [11]. Имеется и ограничение на $s$ сверху. Для больших значений $s$ надо при интегрировании статистической суммы системы учитывать поправку на усреднение фурье-образа потенциала взаимодействия, которая увеличивается с ростом $s$.

В приближении модели $\rho^{6}$ в работе [96] предложен новый метод расчета на микроскопическом уровне уравнения состояния трехмерной изинговской системы, не потерявшего в последнее время несомненного научного интереса (см., например, [97], где уравнение состояния для систем класса универсальности трехмерной модели Изинга исследуется в рамках теоретико-полевого подхода с использованием технических приемов РГ). В пользу избранной для исследования модели $\rho^{6}$ свидетельствует и работа [98], в которой изучается эффективный потенциал для скалярной теории поля в трех размерностях в симметрической фазе. В этой работе указано на необходимость учета в эффективном потенциале члена с шестой степенью переменной. Вычислены значения универсальных четырех- и шеститочечных констант связи. Безразмерные шести- и восьмиточечные эффективные константы связи с использованием теоретико-полевого РГ-подхода в трех измерениях рассчитываются в трехпетлевом приближении в статье [99]. Работа [100] содержит оценку безразмерной шестерной эффективной константы связи в случае трехмерной модели Изинга в четырехпетлевом приближении.

В рамках модели $\rho^{6}$ разработан способ расчета выражений для термодинамических функций трехмерной изинговской системы с учетом слагаемых, определяюших поправку к скейлингу. Расчеты осушествлены для температур выше [101] и ниже [102] тем-

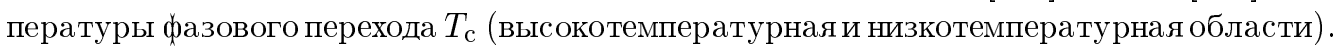
Полученные выражения для ведущих критических амплитуд и амплитуд первой конфлюэнтной поправки позволяют исследовать их зависимость от микроскопических параметров системы (радиуса действия потенциала $b$, постоянной решетки $c$ ). Рассмотрим основные аспекты этих расчетов.

Будем исходить из выражения для статистической суммы в приближении модели $\rho^{6}$. Полагая в $(2.5) n=3$ и осуществляя в $(2.4)$ интегрирование по переменным $\rho_{\mathbf{k}}$ и $\omega_{\mathbf{k}} \mathrm{c}$ индексами $B^{\prime}<k \leqslant B$, а затем еще по $N^{\prime}$ переменным $\omega_{\mathbf{k}}$, получаем

$$
\begin{aligned}
Z= & 2^{N} 2^{\left(N^{\prime}-1\right) / 2} e^{a_{0}^{\prime} N^{\prime}} \int \exp \left[-\frac{1}{2} \sum_{k \leqslant B^{\prime}} d^{\prime}(k) \rho_{\mathbf{k}} \rho_{-\mathbf{k}}-\right. \\
& \left.-\sum_{l=2}^{3} \frac{a_{2 l}^{\prime}}{(2 l) !\left(N^{\prime}\right)^{l-1}} \sum_{k_{1}, \ldots, k_{2 l} \leqslant B^{\prime}} \rho_{\mathbf{k}_{1}} \ldots \rho_{\mathbf{k}_{2 l}} \delta_{\mathbf{k}_{1}+\cdots+\mathbf{k}_{2 l}}\right](d \rho)^{N^{\prime}},
\end{aligned}
$$

где $N^{\prime}=N s_{0}^{-3}, s_{0}=B / B^{\prime}=\pi \sqrt{2} b / c$,

$$
d^{\prime}(k)=a_{2}^{\prime}-\beta \widetilde{\Phi}(k) .
$$


Коэффициенты $a_{2 l}^{\prime}$ определяются соотношениями

$$
\begin{aligned}
& a_{0}^{\prime}=\ln Q(\mathcal{M}), \quad Q(\mathcal{M})=\left(12 s_{0}^{3}\right)^{1 / 4} \pi^{-1} I_{0}\left(\eta^{\prime}, \xi^{\prime}\right), \\
& a_{2}^{\prime}=\left(12 s_{0}^{3}\right)^{1 / 2} F_{2}\left(\eta^{\prime}, \xi^{\prime}\right), \\
& a_{4}^{\prime}=12 s_{0}^{3} C\left(\eta^{\prime}, \xi^{\prime}\right), \\
& a_{6}^{\prime}=\left(12 s_{0}^{3}\right)^{3 / 2} N\left(\eta^{\prime}, \xi^{\prime}\right)
\end{aligned}
$$

и являются функциями $s_{0}$, т.е. отношения $b / c$. Здесь в качестве аргументов выступают величины

$$
\eta^{\prime}=\sqrt{3} s_{0}^{3 / 2}, \quad \xi^{\prime}=\frac{8 \sqrt{3}}{15 s_{0}^{3 / 2}} .
$$

Специальные функции $C\left(\eta^{\prime}, \xi^{\prime}\right)$ и $N\left(\eta^{\prime}, \xi^{\prime}\right)$ имеют вид

$$
\begin{aligned}
& C\left(\eta^{\prime}, \xi^{\prime}\right)=-F_{4}\left(\eta^{\prime}, \xi^{\prime}\right)+3 F_{2}^{2}\left(\eta^{\prime}, \xi^{\prime}\right), \\
& N\left(\eta^{\prime}, \xi^{\prime}\right)=F_{6}\left(\eta^{\prime}, \xi^{\prime}\right)-15 F_{4}\left(\eta^{\prime}, \xi^{\prime}\right) F_{2}\left(\eta^{\prime}, \xi^{\prime}\right)+30 F_{2}^{3}\left(\eta^{\prime}, \xi^{\prime}\right),
\end{aligned}
$$

где

$$
\begin{aligned}
& F_{2 l}\left(\eta^{\prime}, \xi^{\prime}\right)=\frac{I_{2 l}\left(\eta^{\prime}, \xi^{\prime}\right)}{I_{0}\left(\eta^{\prime}, \xi^{\prime}\right)} \\
& I_{2 l}\left(\eta^{\prime}, \xi^{\prime}\right)=\int_{0}^{\infty} t^{2 l} \exp \left(-\eta^{\prime} t^{2}-t^{4}-\xi^{\prime} t^{6}\right) d t .
\end{aligned}
$$

Как показывают расчеты, при $b \gg c$ коэффициенты $a_{2}^{\prime} \rightarrow 1, a_{2 l}^{\prime} \rightarrow 0$ для $l \geqslant 2$ и подынтегральная функция выражения (2.6) по своей форме приближается к гауссову распределению. В случае соразмерности радиуса действия потенциала и постоянной решетки коэффишиенты $a_{2 l}^{\prime}$ для всех значений $l \geqslant 0$ являются ненулевыми и в показателе экспоненты подынтегрального выражения (2.6) необходимо принимать во внимание, кроме гауссовых, слагаемые, пропорциональные высшим степеням переменной $\rho_{\mathbf{k}}$. В дальнейшем будем иметь в виду именно этот случай.

Воспользуемся предложенным в работе [11] методом "послойного" интегрирования (2.6) по переменным $\rho_{\mathbf{k}}$. Интегрирование начинается от переменных $\rho_{\mathbf{k}}$ с большими значениями $k$ (порядка границы полузоны Бриллюэна) и заканчивается $\rho_{\mathbf{k}} \mathrm{c} k \rightarrow 0$. Для этого фазовое пространство КП $\rho_{\mathbf{k}}$ разбивается на слои с параметром деления $s$. В каждом $n$-м слое (соответствуюшая область волновых векторов $B_{n+1}<k \leqslant B_{n}$, $\left.B_{n+1}=B_{n} / s, s>1\right)$ фурье-образ потенциала $\widetilde{\Phi}(k)$ заменяется его средним значением (в данной работе средним арифметическим). Здесь поправку на усреднение потенциала для упрощения изложения принимаем равной нулю, хотя при необходимости ее можно учесть [11]. В результате поэтапного вычисления статистической суммы число переменных интегрирования в выражении для нее постепенно уменьшается, статистическая сумма представляется в виде произведений парциальных статистических сумм отдельных слоев и интеграла от “сглаженной” эффективной плотности меры. После интегрирования по $n+1$ слоям пространства КП получаем

$$
Z=2^{N} 2^{\left(N_{n+1}-1\right) / 2} Q_{0} Q_{1} \ldots Q_{n}\left[Q\left(P_{n}\right)\right]^{N_{n+1}} \int \mathcal{W}_{6}^{(n+1)}(\rho)(d \rho)^{N_{n+1}}
$$




$$
\begin{aligned}
\text { где } N_{n+1} & =N^{\prime} s^{-3(n+1)} \\
Q_{0} & =\left[e^{a_{0}^{\prime}} Q(d)\right]^{N^{\prime}}, \quad Q_{1}=\left[Q(P) Q\left(d_{1}\right)\right]^{N_{1}}, \ldots, Q_{n}=\left[Q\left(P_{n-1}\right) Q\left(d_{n}\right)\right]^{N_{n}} \\
Q\left(d_{n}\right) & =2\left(\frac{24}{a_{4}^{(n)}}\right)^{1 / 4} I_{0}\left(h_{n}, \alpha_{n}\right) \\
Q\left(P_{n}\right) & =\pi^{-1}\left(\frac{s^{3} a_{4}^{(n)}}{C\left(h_{n}, \alpha_{n}\right)}\right)^{1 / 4} I_{0}\left(\eta_{n}, \xi_{n}\right)
\end{aligned}
$$

Основные аргументы

$$
h_{n}=d_{n}\left(B_{n+1}, B_{n}\right)\left(\frac{6}{a_{4}^{(n)}}\right)^{1 / 2}, \quad \alpha_{n}=\frac{\sqrt{6}}{15} \frac{a_{6}^{(n)}}{\left(a_{4}^{(n)}\right)^{3 / 2}}
$$

определяются средним значением коэффишиента $d_{n}(k)$ в $n$-м слое фазового пространства КП, т.е. $d_{n}\left(B_{n+1}, B_{n}\right)$, и величинами $a_{4}^{(n)}, a_{6}^{(n)}$. Эффективная шестерная плотность меры $(n+1)$-й блочной структуры $\mathcal{W}_{6}^{(n+1)}(\rho)$ имеет следуюший вид:

$$
\begin{aligned}
\mathcal{W}_{6}^{(n+1)}(\rho)= & \exp \left[-\frac{1}{2} \sum_{k \leqslant B_{n+1}} d_{n+1}(k) \rho_{\mathbf{k}} \rho_{-\mathbf{k}}-\right. \\
& \left.-\sum_{l=2}^{3} \frac{a_{2 l}^{(n+1)}}{(2 l) ! N_{n+1}^{l-1}} \sum_{k_{1}, \ldots, k_{2 l} \leqslant B_{n+1}} \rho_{\mathbf{k}_{1}} \ldots \rho_{\mathbf{k}_{2 l}} \delta_{\mathbf{k}_{1}+\cdots+\mathbf{k}_{2 l}}\right]
\end{aligned}
$$

Здесь $B_{n+1}=B^{\prime} s^{-(n+1)}, d_{n+1}(k)=a_{2}^{(n+1)}-\beta \widetilde{\Phi}(k), a_{2 l}^{(n+1)}$-перенормированные значения коэффициентов $a_{2 l}^{\prime}$ после интегрирования по $n+1$ слоям фазового пространства КП. Промежуточные переменные $\eta_{n}, \xi_{n}$ являются функциями $h_{n}$ и $\alpha_{n}$. Они задаются выражениями

$$
\begin{aligned}
& \eta_{n}=\left(6 s^{3}\right)^{1 / 2} F_{2}\left(h_{n}, \alpha_{n}\right)\left[C\left(h_{n}, \alpha_{n}\right)\right]^{-1 / 2}, \\
& \xi_{n}=\frac{\sqrt{6}}{15} s^{-3 / 2} N\left(h_{n}, \alpha_{n}\right)\left[C\left(h_{n}, \alpha_{n}\right)\right]^{-3 / 2},
\end{aligned}
$$

где вид специальных функций $C\left(h_{n}, \alpha_{n}\right)$ и $N\left(h_{n}, \alpha_{n}\right)$ определен в $(2.10)$. Коэффициенты $d_{n}\left(B_{n+1}, B_{n}\right), a_{4}^{(n)}, a_{6}^{(n)}$ связаны с коэффициентами $(n+1)$-го слоя РС [93, 103, 104], решения которых $[96,104]$ используются при вычислении свободной энергии системы.

Расчет выражения для вклада в свободную энергию от коротковолновых мод колебаний спиновой плотности связан с суммированием парциальных свободных энергий по слоям фазового пространства КП до точки выхода системы из участка КР [95]. Главной задачей при этом является выделение явной зависимости от номера слоя. Для этой цели используются решения РС. Учет в них большего собственного значения $\left(E_{1}>1\right)$

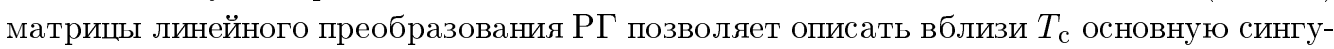
лярность теплоемкости. Меньшие собственные значения $\left(E_{2}<1, E_{3}<1\right)$ отвечают за возникновение поправок к скейлингу. Для учета вклада длинноволновых мод колебаний в свободную энергию системы развит прямой метод расчета. Исходными данными для него являются результаты, полученные при учете коротковолновых мод. 
Подчеркнем, что проводимые расчеты справедливы в узкой окрестности $T_{\mathrm{c}}(|\tau|<$ $\left.\tau^{*} \sim 10^{-2}, \tau=\left(T-T_{\mathrm{c}}\right) / T_{\mathrm{c}}\right)$. Размер этой критической области позволяют оценить решения РС. Действительно, используя эти решения и условие существования КР (выход из него при $n \rightarrow 1$, описываемый слагаемыми, пропорциональными $E_{1}^{n}$, не превьшает входа в КР, описываемого слагаемыми, пропорциональными $\left.E_{2}^{n}, E_{3}^{n}\right)$, можно определить область температур $|\tau|<\tau^{*}$, в которой имеет место КР, соответствующий наличию сильнокоррелированных спиновых блоков. Величина $\tau^{*}$ будет равна меньшему по модулю из корней трех уравнений, полученных на основе решений $\mathrm{PC}$ (см. табл. 1). В табл. 1 величина $b=b_{\mathrm{I}}=c /(2 \sqrt{3})$ соответствует взаимодействию ближайших соседей, $b=b_{\mathrm{II}}=0.3379 c-$ взаимодействию первых и вторых соседей, $b=b_{\mathrm{III}}=0.3584 c-$ первых, вторых и третьих соседей [105].

Таблица 1

\begin{tabular}{|c|c|c|c|c|c|}
\hline & $b_{\mathrm{I}}$ & $b_{\mathrm{II}}$ & $b_{\mathrm{III}}$ & $b_{\mathrm{IV}}=0.5 c$ & $b_{\mathrm{V}}=c$ \\
\hline$\tau^{*}$ & 0.0185 & 0.0222 & 0.0238 & 0.0330 & 0.0421 \\
\hline
\end{tabular}

Характерной особенностью решений РС является специфическая зависимость одного из их коэффициентов от температуры $[101,104,106]$. На основе выражения для этого коэффишиента можно получить уравнение для величины $\beta_{\mathrm{c}} \widetilde{\Phi}(0)$, определяюшей температуру фазового перехода [104]. Зависимость $\beta_{\mathrm{c}} A$ ( $A$ - постоянная, входяшая в потенциал взаимодействия (2.2)) от отношения радиуса действия потенциала $b$ к постоянной решетки $c$ показана на рис. 1. Здесь и в дальнейшем графический материал приводится для параметра РГ $s=3$.

Рассчитав отдельно вклады в свободную энергию системы от коротковолновых $F_{\mathrm{KP}}$ и длинноволновых $F_{\text {ПгР }}\left(T>T_{\mathrm{c}}\right)$ или $F_{\text {ИГр }}\left(T<T_{\mathrm{c}}\right)$ мод колебаний спиновой плотности, можно найти полное выражение для свободной энергии системы

$$
F=F_{0}+F_{\mathrm{KP}}+F_{\text {ПГР }}
$$

в высокотемпературной области или

$$
F=F_{0}+F_{\mathrm{KP}}+F_{\text {ИГР }}
$$

в низкотемпературной. Здесь $F_{0}=-k T N \ln 2-$ свободная энергия $N$ невзаимодействующих спинов.

Методика расчетов подобна методике, разработанной в цикле препринтов $[86,107$ 109], где свободная энергия и другие термодинамические характеристики трехмерной модели Изинга вычисляются с использованием четверной плотности меры. Случаи $T>T_{\text {с и }} T<T_{\text {с }}$ без учета конфлюэнтных поправок рассмотрены соответственно в [86] и [107], с учетом первой конфлюэнтной поправки - в [108] и [109]. Расчет второй конфлюэнтной поправки осушествлен в работе [110]. Работа [111] содержит оценку вклада от этих скейлинговых поправок в теплоемкость системы, которая как функция температуры и микроскопических параметров приведена в [112]. 


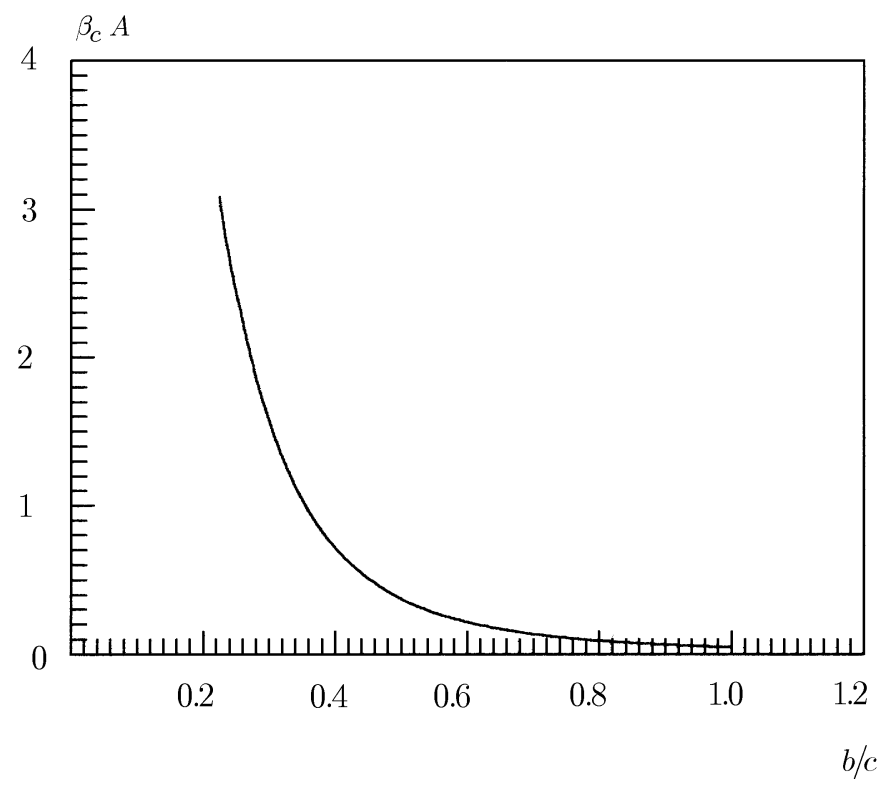

Рис. 1. Зависимость обратной температуры фазового перехода от отношения радиуса действия потенциала взаимодействия к постоянной решетки.

Расчеты вкладов в свободную энергию системы от коротко- и длинноволновых мод колебаний плотности спинового момента и полных выражений для термодинамических функций на основе шестерной плотности меры подробно изложены в $[95,101,102,106]$, причем в $[95,106]$ без учета конфлююэнтных поправок, в $[101,102]$ с учетом первой конффлюэнтной поправки. Ниже приведены окончательные выражения для термодинамических характеристик изинговской системы в приближении модели $\rho^{6}$ с учетом первой конфлюэнтной поправки.

\section{3. ТЕРМОДИНАМИЧЕСКИЕ ХАРАКТЕРИСТИКИ ТРЕХМЕРНОЙ МОДЕЛИ ИЗИНГА КАК ФУНКЦИИ ТЕМПЕРАТУРЫ И МИКРОСКОПИЧЕСКИХ ПАРАМЕТРОВ СИСТЕМЫ}

Сначала рассмотрим высокотемпературную, а затем низкотемпературную области. Первая конфлюэнтная поправка определяется слагаемым, пропорциональным $\tau^{\Delta_{1}}$ $\left(T>T_{\mathrm{c}}\right)$ или $|\tau|^{\Delta_{1}}\left(T<T_{\mathrm{c}}\right)$, где показатель $\Delta_{1}=-\ln E_{2} / \ln E_{1}$. В данных расчетах не принимается во внимание вторая конфлюэнтная поправка (соответствуюшее слагаемое пропорционально $\tau^{\Delta_{2}}\left(T>T_{\mathrm{c}}\right)$ или $\left.\mid \tau^{\Delta_{2}}\left(T<T_{\mathrm{c}}\right), \Delta_{2}=-\ln E_{3} / \ln E_{1}\right)$. Это связано

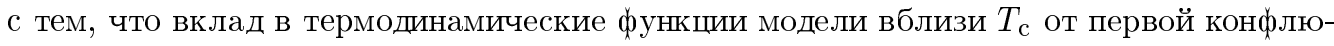
энтной поправки является более сушественным по сравнению с незначительным вкладом от второй поправки $\left(|\tau| \ll 1, \Delta_{1} \sim 0.5, \Delta_{2}>2[95]\right)$. В случае модели $\rho^{4}$ имеем $\Delta_{1}=\Delta=-\ln E_{2} / \ln E_{1}, \Delta_{2}=2 \Delta, \Delta \sim 0.5$ [88].

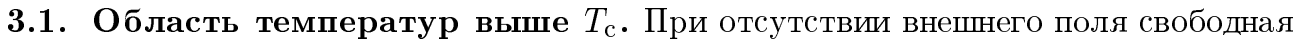


Таблица 2

\begin{tabular}{|c|c|c|c|c|c|}
\hline Коэффициенты & $b_{\mathrm{I}}$ & $b_{\mathrm{II}}$ & $b_{\mathrm{III}}$ & $b_{\mathrm{IV}}=c$ & $b_{\mathrm{V}}=2 c$ \\
\hline & \multicolumn{5}{|c|}{$s=2.0000$} \\
\hline$\gamma_{0}$ & 1.8758 & 2.7464 & 3.1962 & 61.1798 & 486.699 \\
\hline$\gamma_{1}$ & -0.8032 & -0.7759 & -0.7651 & -0.6734 & -0.6701 \\
\hline \multirow[t]{2}{*}{$\gamma_{2}$} & -4.4816 & -3.9551 & -3.7548 & -2.0482 & -1.9599 \\
\hline & \multicolumn{5}{|c|}{$s=2.7349$} \\
\hline$\gamma_{0}$ & 1.8776 & 2.7496 & 3.2000 & 61.1930 & 486.713 \\
\hline$\gamma_{1}$ & -0.7063 & -0.6952 & -0.6913 & -0.6924 & -0.6978 \\
\hline \multirow[t]{2}{*}{$\gamma_{2}$} & -4.6948 & -4.1735 & -3.9764 & -2.2672 & -2.1665 \\
\hline & \multicolumn{5}{|c|}{$s=3.0000$} \\
\hline$\gamma_{0}$ & 1.8789 & 2.7516 & 3.2023 & 61.1999 & 486.720 \\
\hline$\gamma_{1}$ & -0.6867 & -0.6795 & -0.6773 & -0.7020 & -0.7100 \\
\hline$\gamma_{2}$ & -4.5304 & -4.0342 & -3.8466 & -2.1971 & -2.0936 \\
\hline
\end{tabular}

энергия системы согласно (2.17) принимает вид [101]

$$
\begin{gathered}
F=-k T N^{\prime}\left[\gamma_{0}+\gamma_{1} \tau+\gamma_{2} \tau^{2}+\gamma_{3}^{(0)+} \tau^{3 \nu}+\gamma_{3}^{(1)+} \tau^{3 \nu+\Delta_{1}}\right] \\
\gamma_{3}^{(l)+}=c_{\nu}^{3} c_{\Delta_{1}}^{l} \bar{\gamma}_{3}^{(l)+}, \quad l=0,1 .
\end{gathered}
$$

Здесь $\nu=\ln s / \ln E_{1}$ - критический показатель корреляционной длины. Слагаемые, пропорциональные целым степеням $\tau$ в $(3.1)$, появляются исключительно за счет учета коротковолновых мод колебаний. Члены, пропорциональные $\tau^{3 \nu}, \tau^{3 \nu+\Delta_{1}}$ (неаналитическая часть свободной энергии), формируются в результате учета как коротко-, так и длинноволновых мод колебаний. Причем первая конфлюэнтная поправка возникает за счет учета в решениях $\mathrm{PC}$ меньшего собственного значения $E_{2}$ матрицы линейного преобразования РГ.

Все коэффициенты в (3.1) являются функциями микроскопических параметров системы, т.е. радиуса действия потенциала $b$, фурье-образа потенциала $\tilde{\Phi}(0)$ при $k=0$ и постоянной решетки $c$. Основным достоинством выражения для свободной энергии $F$ является наличие соотношений, связьваюших ее коэффициенты с микроскопически- 
ми параметрами системы и координатами фиксированной точки РС. Значения коэффициентов $\gamma_{0}, \gamma_{1}, \gamma_{2}$ [101] не зависят от того, выше или ниже температуры фазового перехода осушествляются расчеты. Они приведены в табл. 2. Численные расчеты даются для разных значений параметра $s$ и радиуса эффективного действия потенциала $b$. Отметим, что при $s=s^{*}=2.7349$ значение основной переменной в фиксированной точке $h^{(0)}$ примерно равно нулю [113] (для модели $\rho^{4}$ соответствуюшее значение $\left.s^{*}=3.5862[11,86]\right)$. Значения $s$, близкие к $s^{*}$, являются оптимальными для данной методики вычислений.

Таблица 3

\begin{tabular}{|c|c|c|}
\hline$s$ & $\bar{\gamma}_{3}^{(0)+}$ & $\bar{\gamma}_{3}^{(1)+}$ \\
\hline 2.0000 & 0.9699 & 0.6508 \\
2.7349 & 1.8654 & 0.7263 \\
3.0000 & 2.1770 & 0.7162 \\
\hline
\end{tabular}

Как видим из $(3.1)$, коэффициенты $\gamma_{3}^{(l)+}(l=0,1)$ имеют форму произведения универсальной по отношению к микроскопическим параметрам величины $\bar{\gamma}_{3}^{(l)+}$ (табл. 3) и неуниверсального множителя $c_{\nu}^{3} c_{\Delta_{1}}^{l}$ [101], зависящего от них. Аналогичными соотношениями определяются основные критические амплитуды и амплитуды конфлюэнтной поправки других термодинамических характеристик системы. Их отношение при $T>T_{\mathrm{c}}$

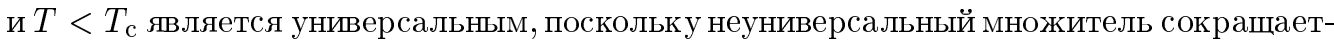
ся.

Исходя из свободной энергии (3.1) можно найти энтропию, внутреннюю энергию и теплоемкость системы. Их коэффициенты будут выражаться через коэффициенты свободной энергии. Для энтропии $S$, внутренней энергии $U$ и теплоемкости $C$ получаем

$$
\begin{aligned}
S & =k N^{\prime}\left[s^{(0)}+c_{0} \tau+u_{3}^{(0)+} \tau^{1-\alpha}+u_{3}^{(1)+} \tau^{1-\alpha+\Delta_{1}}\right] \\
U & =k T N^{\prime}\left[\gamma_{1}+u_{1} \tau+u_{3}^{(0)+} \tau^{1-\alpha}+u_{3}^{(1)+} \tau^{1-\alpha+\Delta_{1}}\right], \\
C & =k N^{\prime}\left[c_{0}+c_{3}^{(0)+} \tau^{-\alpha}+c_{3}^{(1)+} \tau^{\Delta_{1}-\alpha}\right]
\end{aligned}
$$

где

$$
\begin{aligned}
s^{(0)} & =\gamma_{0}+\gamma_{1}, \\
c_{0} & =2\left(\gamma_{1}+\gamma_{2}\right), \\
u_{1} & =\gamma_{1}+2 \gamma_{2}, \\
u_{3}^{(l)+} & =c_{\nu}^{3} c_{\Delta_{1}}^{l} \bar{u}_{3}^{(l)+}, \quad l=0,1, \\
\bar{u}_{3}^{(0)+} & =3 \nu \bar{\gamma}_{3}^{(0)+}, \\
\bar{u}_{3}^{(1)+} & =\left(3 \nu+\Delta_{1}\right) \bar{\gamma}_{3}^{(1)+}, \\
c_{3}^{(l)+} & =c_{\nu}^{3} c_{\Delta_{1}}^{l} \bar{c}_{3}^{(l)+},
\end{aligned}
$$




$$
\begin{aligned}
& \bar{c}_{3}^{(0)+}=3 \nu(3 \nu-1) \bar{\gamma}_{3}^{(0)+}, \\
& \bar{c}_{3}^{(1)+}=\left(3 \nu+\Delta_{1}\right)\left(3 \nu+\Delta_{1}-1\right) \bar{\gamma}_{3}^{(1)+},
\end{aligned}
$$

здесь $\alpha=2-3 \nu-$ критический показатель теплоемкости.

\begin{tabular}{|c|c|c|c|c|c|}
\hline Коэффициенты & $b_{\mathrm{I}}$ & $b_{\text {II }}$ & $b_{\text {III }}$ & $b_{\mathrm{IV}}=c$ & $b_{\mathrm{V}}=2 c$ \\
\hline & \multicolumn{5}{|c|}{$s=2.0000$} \\
\hline$A^{+}$ & 1.0876 & 0.9960 & 0.9609 & 0.6620 & 0.6471 \\
\hline$a_{c}^{+}$ & -1.2609 & -1.8262 & -2.0389 & -3.7773 & -3.8634 \\
\hline$B^{+}$ & -10.5696 & -9.4620 & -9.0397 & -5.4430 & -5.2601 \\
\hline$\Gamma^{+}$ & 1.8711 & 1.9842 & 2.0321 & 2.6052 & 2.6450 \\
\hline \multirow[t]{2}{*}{$a_{\chi}^{+}$} & -0.0691 & -0.1001 & -0.1118 & -0.2071 & -0.2118 \\
\hline & \multicolumn{5}{|c|}{$s=2.7349$} \\
\hline$A^{+}$ & 0.8113 & 0.7439 & 0.7184 & 0.5050 & 0.4944 \\
\hline$a_{c}^{+}$ & -2.3816 & -2.7420 & -2.8773 & -3.9838 & -4.0397 \\
\hline$B^{+}$ & -10.8022 & -9.7375 & -9.3355 & -5.9193 & -5.7286 \\
\hline$\Gamma^{+}$ & 2.1659 & 2.2948 & 2.3488 & 2.9709 & 3.0134 \\
\hline \multirow[t]{2}{*}{$a_{\chi}^{+}$} & -0.1177 & -0.1355 & -0.1422 & -0.1969 & -0.1996 \\
\hline & \multicolumn{5}{|c|}{$s=3.0000$} \\
\hline$A^{+}$ & 0.7238 & 0.6644 & 0.6420 & 0.4558 & 0.4465 \\
\hline$a_{c}^{+}$ & -2.6494 & -2.9650 & -3.0832 & -4.0460 & -4.0947 \\
\hline$B^{+}$ & -10.4343 & -9.4274 & -9.0478 & -5.7981 & -5.6074 \\
\hline$\Gamma^{+}$ & 2.4427 & 2.5860 & 2.6459 & 3.3248 & 3.3710 \\
\hline$a_{\chi}^{+}$ & -0.1291 & -0.1445 & -0.1502 & -0.1971 & -0.1995 \\
\hline
\end{tabular}

Таблиша 4 
Формулу для теплоемкости (см. (3.2)) исследуемой модели можно переписать в другом виде $[114,115]$, а именно:

$$
\begin{aligned}
\frac{C}{k N^{\prime}} & =\frac{A^{+}}{\alpha} \tau^{-\alpha}\left(1+\alpha a_{c}^{+} \tau^{\Delta_{1}}\right)+B^{+}, \\
A^{+} & =c_{\nu}^{3} \alpha \bar{c}_{3}^{(0)+}, \quad a_{c}^{+}=\frac{c_{\Delta_{1}}}{\alpha} \frac{\bar{c}_{3}^{(1)+}}{\bar{c}_{3}^{(0)+}}, \quad B^{+}=c_{0} .
\end{aligned}
$$

Такая важная характеристика системы, как восприимчивость на одну частицу при бесконечно малых значениях внешнего поля вблизи $T_{\mathrm{c}}$, задается выражением

$$
\chi=\Gamma^{+} \tau^{-\gamma}\left(1+a_{\chi}^{+} \tau^{\Delta_{1}}\right) \frac{\mu_{\mathrm{Б}}^{2}}{\widetilde{\Phi}(0)},
$$

где $\gamma=2 \nu$ - критический показатель восприимчивости, $\mu_{\mathrm{Б}}$ - магнетон Бора. Выражения для амплитуд $\Gamma^{+}, a_{\chi}^{+}$приведены в работе [101]. Рассчитанные для разных значений $b$ в приближении модели $\rho^{6}$ коэффициенты теплоемкости $C / k N^{\prime}(3.4)$ и восприимчивости $\chi(3.5)$ содержатся в табл. 4.

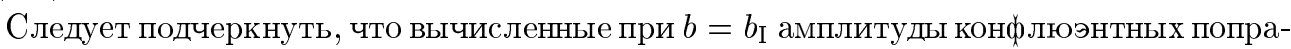
вок $a_{c}^{+}, a_{\chi}^{+}$согласуются с результатами работы [54], где ведущие поправки к скейлинговым амплитудам рассматриваются для моделей Изинга со взаимодействием между ближайшими соседями на простой кубической, ОЦК- и ГЦК-решетках. В [54] показано, что амплитуды указанных поправок для восприимчивости, коррелящионной длины, теплоемкости и спонтанной намагниченности имеют отрицательный знак для всех трех типов решеток. Там же отмечается согласие полученных результатов с результатами высокотемпературного разложения и данными теоретико-полевого анализа.

3.2. Область температур ниже $T_{\mathrm{c}}$. В случае нулевого внешнего поля для полной свободной энергии трехмерной модели Изинга при $T<T_{\mathrm{c}}$ имеем [102]

$$
F=-k T N^{\prime}\left[\gamma_{0}-\gamma_{1}|\tau|+\gamma_{2}|\tau|^{2}+\gamma_{3}^{(0)-}|\tau|^{3 \nu}+\gamma_{3}^{(1)-}|\tau|^{3 \nu+\Delta_{1}}\right] .
$$

Здесь коэффициенты $\gamma_{0}, \gamma_{1}, \gamma_{2}$ такие же, как и в (3.1) (см. табл. 2). Численные значения универсальной части $\bar{\gamma}_{3}^{(l)-}$ коэффициентов

$$
\gamma_{3}^{(l)-}=c_{\nu}^{3} c_{\Delta_{1}}^{l} \bar{\gamma}_{3}^{(l)-}, \quad l=0,1
$$

приведены в табл. 5 .

Таблица 5

\begin{tabular}{|c|c|c|}
\hline$s$ & $\bar{\gamma}_{3}^{(0)-}$ & $\bar{\gamma}_{3}^{(1)-}$ \\
\hline 2.0000 & 1.7599 & -6.7968 \\
2.7349 & 2.7650 & -3.6743 \\
3.0000 & 3.1073 & -3.0714 \\
\hline
\end{tabular}


Для энтропии, внутренней энергии и теплоемкости системы справедливы выражения

$$
\begin{aligned}
S & =k N^{\prime}\left[s^{(0)}-c_{0}|\tau|-u_{3}^{(0)-}|\tau|^{1-\alpha}-u_{3}^{(1)-}|\tau|^{1-\alpha+\Delta_{1}}\right] \\
U & =k T N^{\prime}\left[\gamma_{1}-u_{1}|\tau|-u_{3}^{(0)-}|\tau|^{1-\alpha}-u_{3}^{(1)-}|\tau|^{1-\alpha+\Delta_{1}}\right] \\
C & =k N^{\prime}\left[c_{0}+c_{3}^{(0)-}|\tau|^{-\alpha}+c_{3}^{(1)-}|\tau|^{\Delta_{1}-\alpha}\right]
\end{aligned}
$$

где $s^{(0)}, c_{0}, \gamma_{1}, u_{1}$ совпадают с соответствуюшими величинами при $T>T_{\mathrm{c}}[101]$, а структура остальных коэффициентов в плане универсальности определяется соотношениями, подобными (3.3).

Для среднего спинового момента, играющего роль параметра порядка исследуемой системы, получаем [102]

$$
\langle\sigma\rangle=\langle\sigma\rangle^{(0)}|\tau|^{\beta}\left(1+\langle\sigma\rangle^{(1)}|\tau|^{\Delta_{1}}\right) .
$$

\begin{tabular}{|c|c|c|c|c|c|}
\hline Коэфффициенты & $b_{\mathrm{I}}$ & $b_{\text {II }}$ & $b_{\text {III }}$ & $b_{\mathrm{IV}}=c$ & $b_{\mathrm{V}}=2 c$ \\
\hline & \multicolumn{5}{|c|}{$s=2.0000$} \\
\hline$\langle\sigma\rangle^{(0)}$ & 2.7329 & 2.0684 & 1.8700 & 0.3747 & 0.1321 \\
\hline \multirow[t]{2}{*}{$\langle\sigma\rangle^{(1)}$} & 0.2499 & 0.3619 & 0.4040 & 0.7485 & 0.7656 \\
\hline & \multicolumn{5}{|c|}{$s=2.7349$} \\
\hline$\langle\sigma\rangle^{(0)}$ & 2.3854 & 1.8027 & 1.6288 & 0.3248 & 0.1145 \\
\hline \multirow[t]{2}{*}{$\langle\sigma\rangle^{(1)}$} & 0.3034 & 0.3493 & 0.3666 & 0.5076 & 0.5147 \\
\hline & \multicolumn{5}{|c|}{$s=3.0000$} \\
\hline$\langle\sigma\rangle^{(0)}$ & 2.2861 & 1.7269 & 1.5600 & 0.3107 & 0.1095 \\
\hline$\langle\sigma\rangle^{(1)}$ & 0.3046 & 0.3409 & 0.3545 & 0.4651 & 0.4707 \\
\hline
\end{tabular}

Здесь $\beta=\nu / 2$ - критический показатель среднего спинового момента, коэффициенты $\langle\sigma\rangle^{(l)}$ для некоторых значений радиуса действия потенциала $b$ и параметра РГ $s$ содержатся в табл. 6.

Кривые зависимости $\langle\sigma\rangle$ от $\tau$ для разных значений $b$ показаны на рис. 2.

Выражения для основных критических амплитуд и амплитуд поправки к скейлингу теплоемкости

$$
\frac{C}{k N^{\prime}}=\frac{A^{-}}{\alpha}|\tau|^{-\alpha}\left(1+\alpha a_{c}^{-}|\tau|^{\Delta_{1}}\right)+B^{-}
$$


$\langle\sigma\rangle$
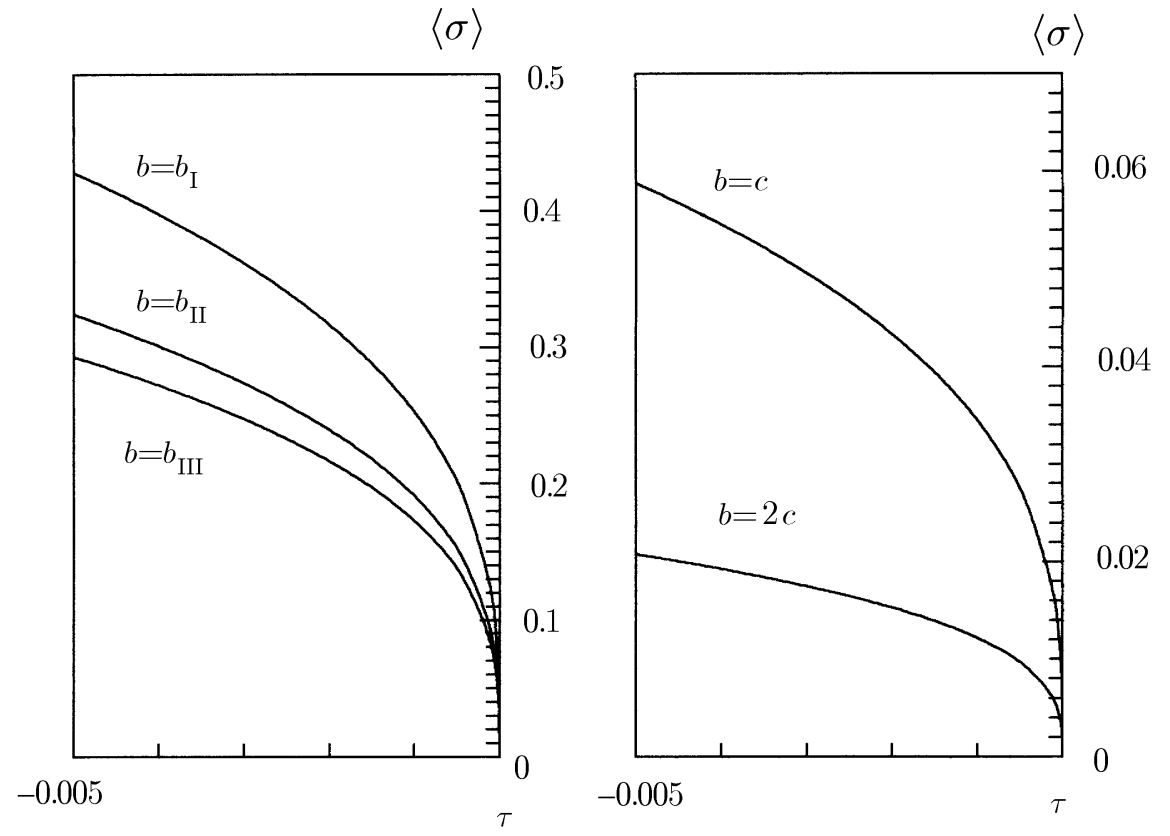

Рис. 2. Температурная зависимость среднего спинового момента в рамках модели $\rho^{6}$ для различных значений радиуса действия потенциала $b: \quad b=b_{\mathrm{I}}=c /(2 \sqrt{3}), \quad b=b_{\mathrm{II}}=$ $0.3379 c, b=b_{\mathrm{III}}=0.3584 c$.

и восприимчивости системы на одну частищу

$$
\chi=\Gamma^{-}|\tau|^{-\gamma}\left(1+a_{\chi}^{-}|\tau|^{\Delta_{1}}\right) \frac{\mu_{\mathrm{5}}^{2}}{\tilde{\Phi}(0)}
$$

приведены в работе [102]. Значения этих амплитуд для разных $b$ представлены в табл. 7. Заметим, что $B^{-}=B^{+}$при $T>T_{\text {с }}$ (см. табл. 4 ).

На основе результатов вычислений в высокотемпературной и низкотемпературной областях можно получить графики температурных зависимостей энтропии $S / k N$, теплоемкости $C / k N$ и восприимчивости $\chi$ (в единицах $\mu_{\mathrm{Б}}^{2} / A$, где $A=\tilde{\Phi}(0) /\left[8 \pi(b / c)^{3}\right]-$

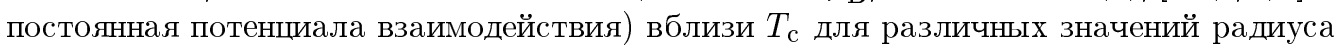
действия потенциала $b$ (см. рис. $3-5)$.

Развитая методика расчета позволяет проследить поведение термодинамических характеристик с ростом отношения радиуса действия потеншиала $b$ к постоянной решетки $c$. Графики свободной энергии системы $F / N$ (в единицах $A$ ) в точке фазового перехода $(\tau=0)$, а также среднего спинового момента $\langle\sigma\rangle$ при $\tau=-10^{-3}$ и теплоемкости системы $C / k N$ при $|\tau|=10^{-3}$ изображены соответственно на рис. 6-8.

Таким образом, статистическое описание основных свойств однокомпонентной спиновой системы с учетом ее микроскопических параметров рассмотрено схематически на 


\begin{tabular}{|c|c|c|c|c|c|}
\hline \multicolumn{5}{|c|}{ Таблица 7 } \\
\hline$A^{-}$ & 1.9734 & 1.8071 & 1.7436 & 1.2012 & 1.1741 \\
$a_{c}^{-}$ & 7.2567 & 10.5104 & 11.7347 & 21.7395 & 22.2353 \\
$\Gamma^{-}$ & 0.2133 & 0.2262 & 0.2317 & 0.2970 & 0.3015 \\
$a_{\chi}^{-}$ & 0.1872 & 0.2711 & 0.3027 & 0.5608 & 0.5736 \\
& & & $s=2.7349$ & & \\
$A^{-}$ & 1.2026 & 1.1027 & 1.0648 & 0.7486 & 0.7328 \\
$a_{c}^{-}$ & 8.1288 & 9.3588 & 9.8206 & 13.5975 & 13.7882 \\
$\Gamma^{-}$ & 0.2341 & 0.2480 & 0.2539 & 0.3211 & 0.3257 \\
$a_{\chi}^{-}$ & 0.3536 & 0.4071 & 0.4272 & 0.5915 & 0.5998 \\
& & & $s=3.0000$ & & \\
$A^{-}$ & 1.0331 & 0.9484 & 0.9164 & 0.6506 & 0.6373 \\
$a_{c}^{-}$ & 7.9599 & 8.9081 & 9.2633 & 12.1558 & 12.3022 \\
$\Gamma_{\overline{\mathrm{III}}}^{-}$ & 0.2437 & 0.2580 & 0.2640 & 0.3318 & 0.3364 \\
\hline & 0.3884 & 0.4346 & 0.4519 & 0.5931 & 0.6002 \\
\hline
\end{tabular}

основе шестерной плотности меры (модель $\left.\rho^{6}\right)$. Это описание в приближении указанной модели $\rho^{6}$ согласуется с результатами расчетов для модели $\rho^{4}$ и данными других авторов (см., например, [95]).

На рис. 9 и 10 показаны графики температурных зависимостей соответственно среднего спинового момента $\langle\sigma\rangle$ и теплоемкости $C / k N$ трехмерной модели Изинга. Вычисления выполнены при отсутствии внешнего поля для случая простой кубической решетки и взаимодействия ближайших соседей. В этих расчетах полагалось, что $b=$ $b_{\mathrm{I}}=c /(2 \sqrt{3})$. При таком $b$ и при $\widetilde{\Phi}(0)=6 J \quad(J-$ постоянная взаимодействия ближайших соседей) в области малых значений волновых векторов параболическая аппроксимация фурье-образа экспоненциально убываюшего потеншиала взаимодействия отвечает аналогичной аппроксимации фурье-образа потенциала взаимодействия ближайших соседей [105]. Приближение моделью $\rho^{6}$ включает первую конфлюэнтную поправ- 

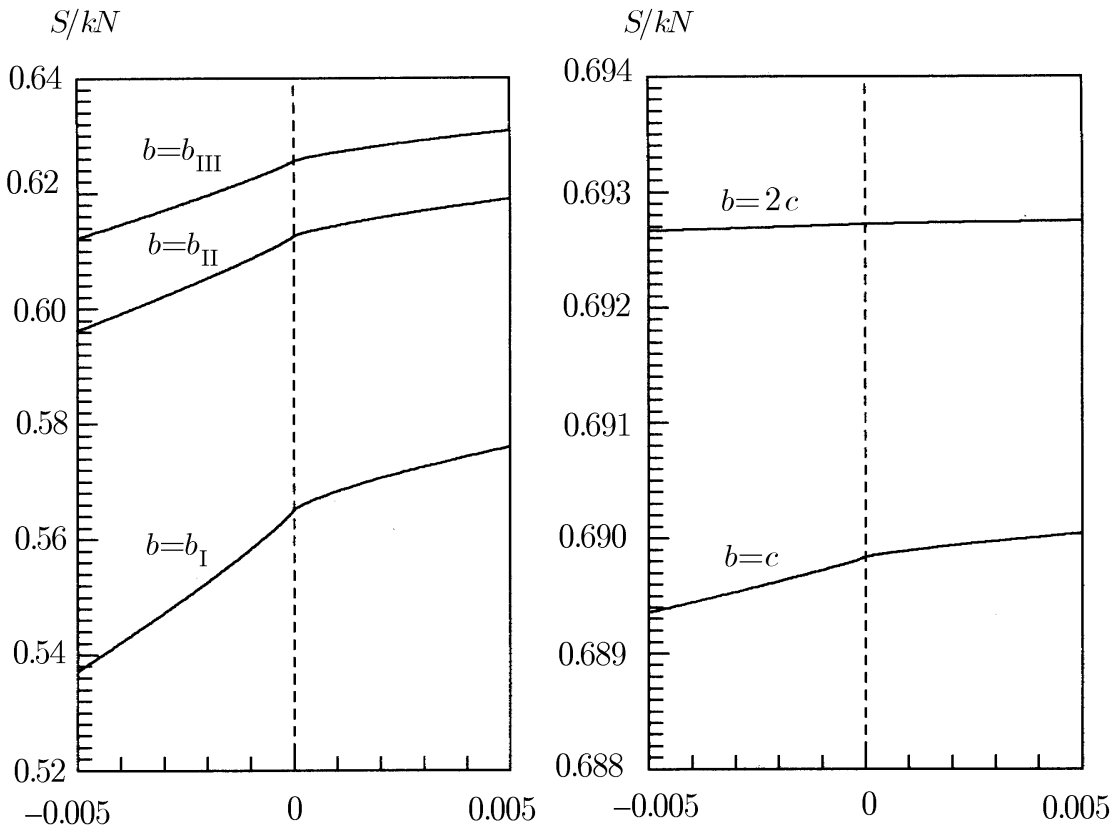

Рис. 3. Зависимость энтропии системы от $\tau$ (обозначения те же, что и на рис. 2 ).

ку $[101,102]$, а моделью $\rho^{4}$ - первую и вторую конффлюэнтные поправки $[88,89,110-112]$.

На рис. 9 линия 1 для среднего спинового момента соответствует модели $\rho^{4}, 2$ - модели $\rho^{6}, 3$ - данным работы [53], $\tau=\left|T-T_{\mathrm{c}}\right| / T_{\mathrm{c}}$. На рис. 10 высокотемпературная область представлена линиями $1,2,3$, а низкотемпературная область - линиями $1^{\prime}, 2^{\prime}, 3^{\prime}$. Линии 1 и $1^{\prime}$ этого рисунка получены с использованием модели $\rho^{4}, 2$ и $2^{\prime}-$ модели $\rho^{6}, 3$ и $3^{\prime}-$ результаты работы [53]. Авторы [53] осушествили новый численный анализ основных критических амплитуд восприимчивости, корреляционной длины, теплоемкости, спонтанной намагниченности трехмерной изинговской системы, универсальных отношений этих амплитуд. Современные оценки критической температуры и показателей здесь использованы вместе с дифференциальными аппроксимантами при экстраполяции имеющихся в распоряжении рядов.

\section{4. ОСНОВНЫЕ РЕЗУЛЬТАТЫ ИССЛЕДОВАНИЯ КРИТИЧЕСКОГО ПОВЕДЕНИЯ ТРЕХМЕРНОЙ МОДЕЛИ ИЗИНГА В РАМКАХ МЕТОДА КОЛЛЕКТИВНЫХ ПЕРЕМЕННЫХ}

Основы метода КП применительно к модели Изинга были заложены в 70-х-80-х годах. В частности, были изучены и решены такие важные вопросы, как вопрос о базисном распределении мод колебаний спиновой плотности в окрестности $T_{\mathrm{c}}$, построение исходного выражения для статистической суммы в виде функционала с явно известными 
$C / k N$

10

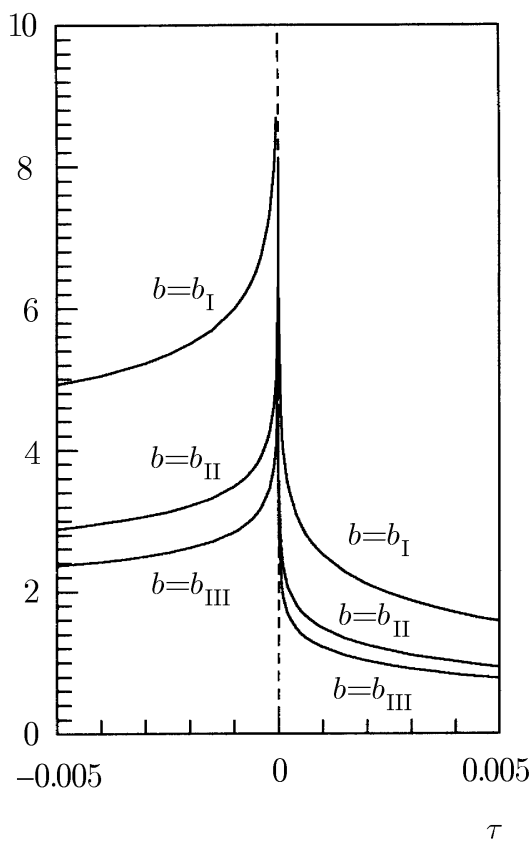

$C / k N$

0.16

0.08

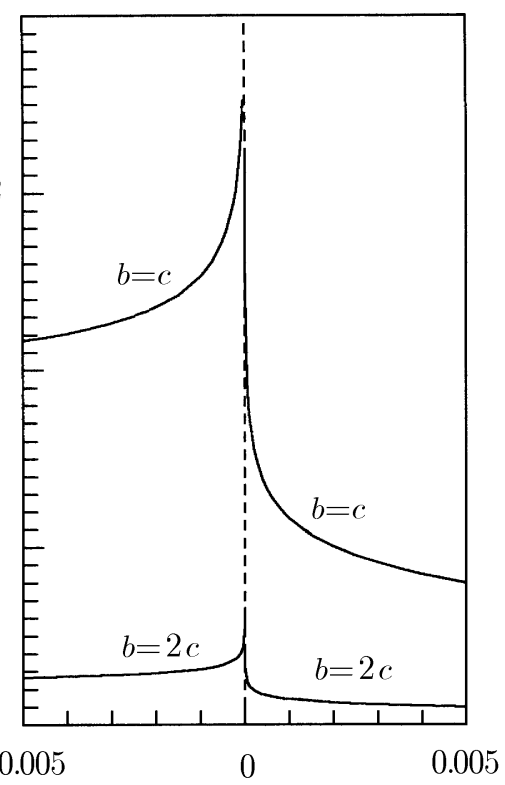

Рис. 4. Теплоемкость спиновой системы при разных значениях $b$ (обозначения те же, что и на рис. 2).

коэффициентными функциями, методика ее расчета, построение разностных уравнений РГ и нахождение их решений $[11,69,85]$. Было показано, что в критической области существует базисная плотность меры, которая, в отличие от гауссовой, содержит в показателе экспоненты вторую и четвертую степени КП. Коэффициенты двух смежных четверных плотностей мер связаны меж ду собой РС, которые вблизи критической температуры допускают решения, позволяюшие произвести расчет коротковолновой части статистической суммы системы.

В представлении КП статистическая сумма трехмерной модели Изинга записывается в виде интеграла по этим КП [85]. При интегрировании по слоям фазового пространства КП используются негауссовы плотности мер, что является важным условием описания поведения системы вблизи точки фазового перехода. Изучено влияние усложнения формы негауссовой плотности меры на критические свойства трехмерной изинговской системы $[11,90-94]$. При этом получены и исследованы общие РС между коэффициентами эффективных плотностей мер, включающих, кроме второй и четвертой, также шестую, восьмую и десятую степени переменной. Представление РС в виде неасимптотических рядов связано с отказом от использования традиционной теории возмущений, основанной на гауссовой плотности меры. Установлено, что начиная с шестерной плотности меры происходит незначительное изменение значения критического показателя корреляционной длины $\nu$, имеющего тенденцию к насышению при последовательном учете 

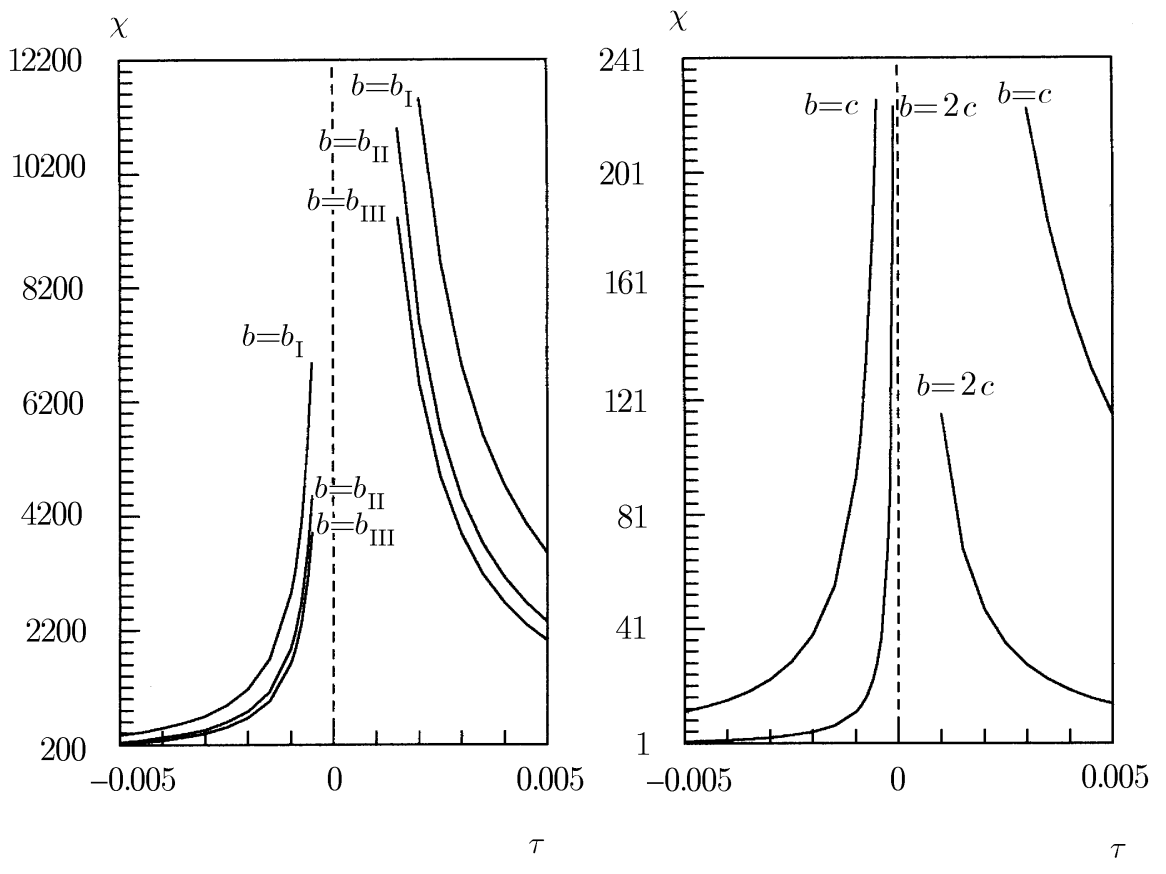

Рис. 5. Температурная зависимость восприимчивости системы для различных значений $b$ (обозначения те же, что и на рис. 2).

негауссовых плотностей мер высшего порядка $\left(\rho^{2 m}\right.$-моделей, $\left.m=3,4,5\right)$.

На основе шестерной плотности меры осушествлено численное исследование статистической суммы трехмерной модели Изинга [116]. С использованием данных численного анализа изучена (вблизи $T_{\mathrm{c}}$ ) эволюция коэффициентов эффективных шестерных плотностей мер с ростом номера слоя фазового пространства КП. Определены размеры коррелирующих блоков для температур, близких к температуре фазового перехода.

Показано, что учет при интегрировании статистической суммы поправки на усреднение фурье-образа потенциала взаимодействия и сдвига при этом фиксированной точки не приводит к качественным изменениям основных термодинамических характеристик системы [117]. Происходит перенормировка критических показателей корреляционной длины, восприимчивости и теплоемкости. Приведены и исследованы возникаюшие при этом РС, найдены перенормированные значения указанных критических показателей. Учет вьшеупомянутой поправки приводит к отличному от нуля значению критического показателя $\eta(\eta \approx 0.024)$, характеризуюшего поведение парной коррелящионной функции при $T=T_{\mathrm{c}}$, выражение для фурье-образа которой в случае четверной плотности меры получено в [118].

Оригинальный метод прямого расчета термодинамических характеристик трехмерной модели Изинга вблизи $T_{\mathrm{c}}$ с учетом конфлюэнтных поправок развит в приближении как четверного [87-89], так и шестерного [101, 102] распределений мод колебаний спино- 


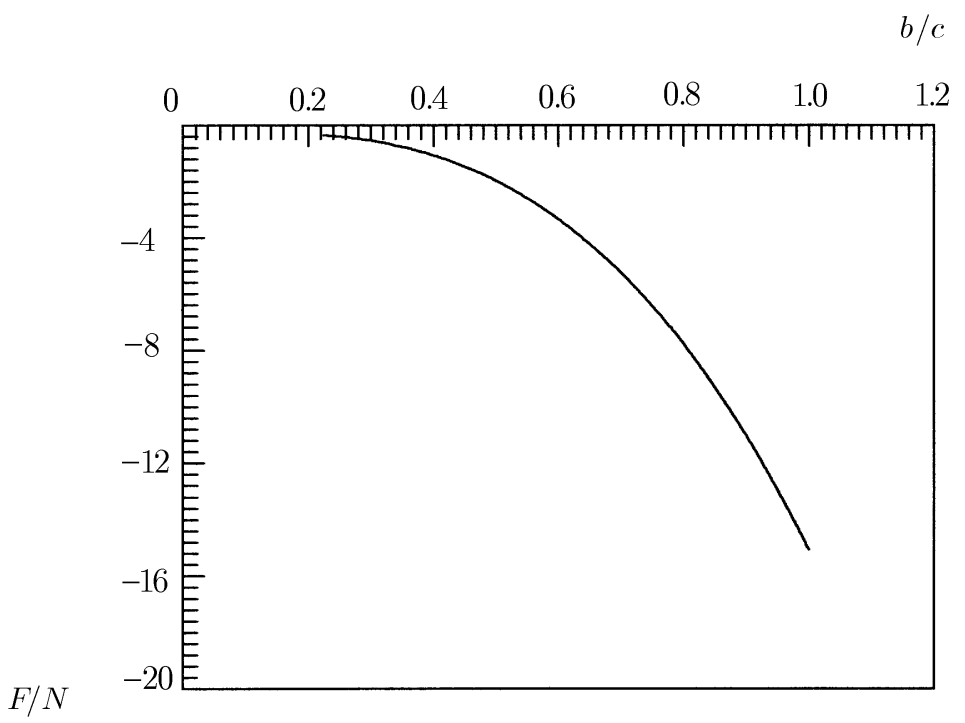

Рис. 6. Зависимость свободной энергии системы в точке фазового перехода $(\tau=0)$ от отношения радиуса действия экспоненциально убывающего потенциала взаимодействия $b$ к постоянной простой кубической решетки $c$.

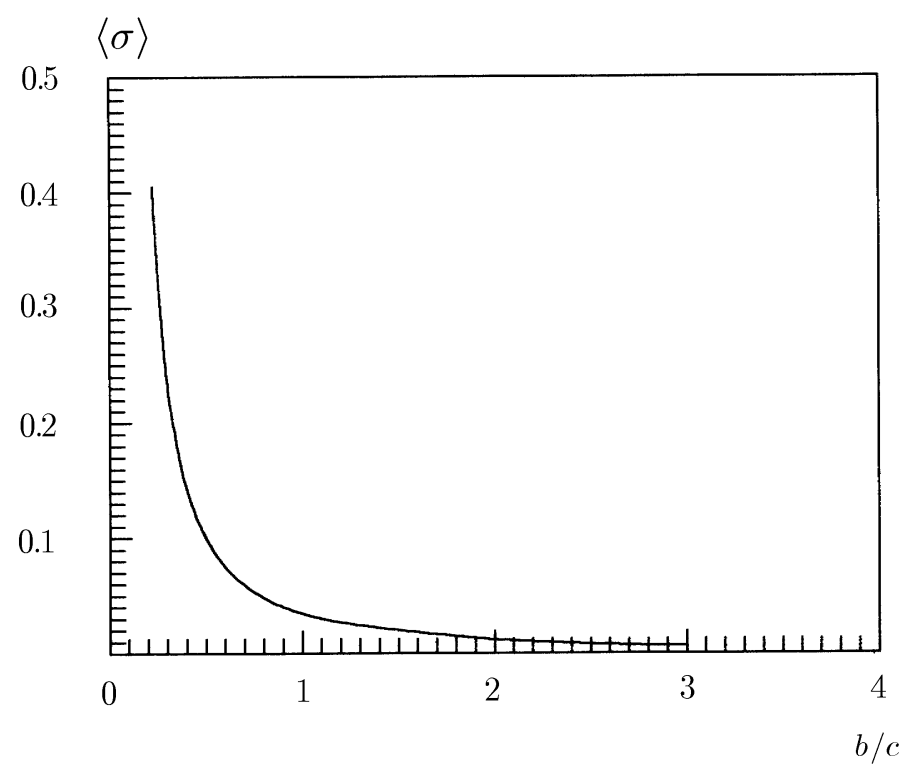

Рис. 7. Зависимость среднего спинового момента при $\tau=-10^{-3}$ от $b / c$.

вой плотности (модели $\rho^{4}$ и $\rho^{6}$, соответственно). Расчеты проиллюстрированы случаем простой кубической решетки и эспоненциально убывающего потенциала взаимодействия. Рассмотрены высокотемпературная $\left(T>T_{\mathrm{c}}\right)$ и низкотемпературная $\left(T<T_{\mathrm{c}}\right)$ области. Свободная энергия, энтропия, внутренняя энергия, теплоемкость, средний спи- 


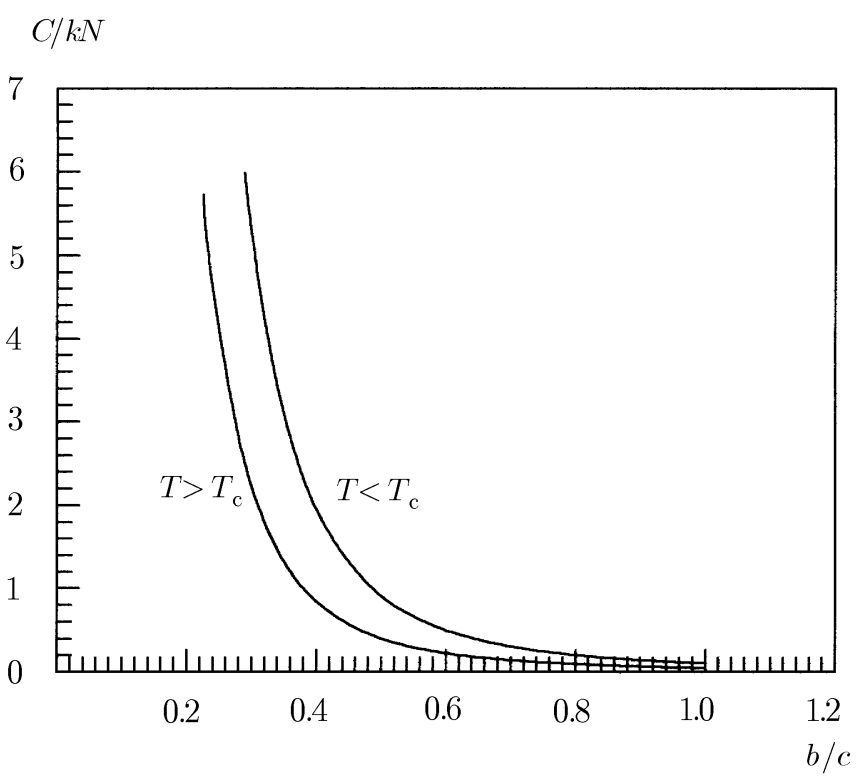

Рис. 8. Зависимость теплоемкости системы при $|\tau|=10^{-3}$ от $b / c$.

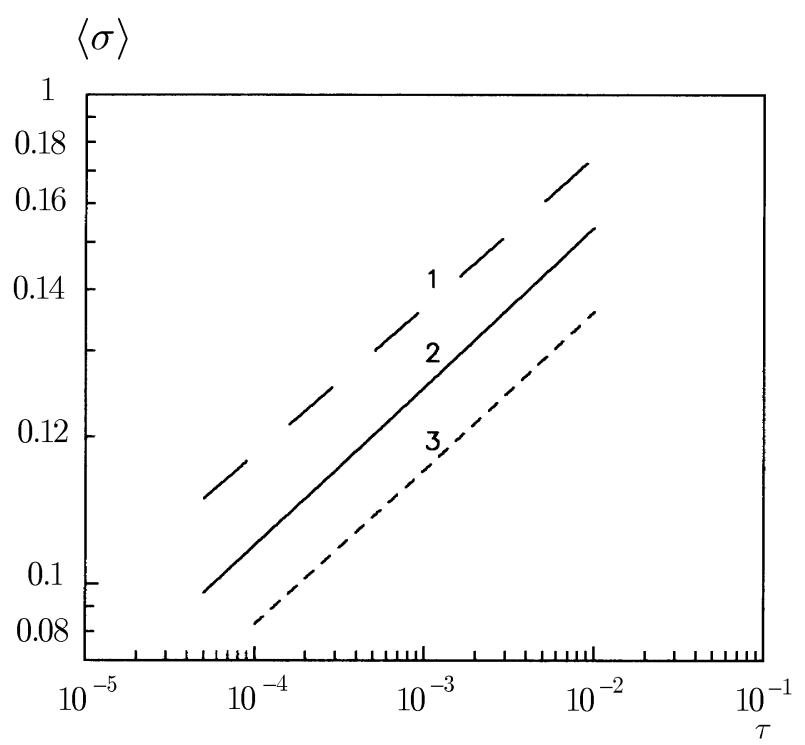

Рис. 9. Температурная зависимость параметра порядка трехмерной модели Изинга на простой кубической решетке.

новый момент и восприимчивость получены как функции температуры и микроскопических параметров системы (радиуса действия потенциала $b$, постоянной решетки $c$ и фурье-образа потенциала $\tilde{\Phi}(0)$ при нулевом значении волнового вектора). В низкотемпературной области вычислен микроскопический аналог свободной энергии Ландау с 


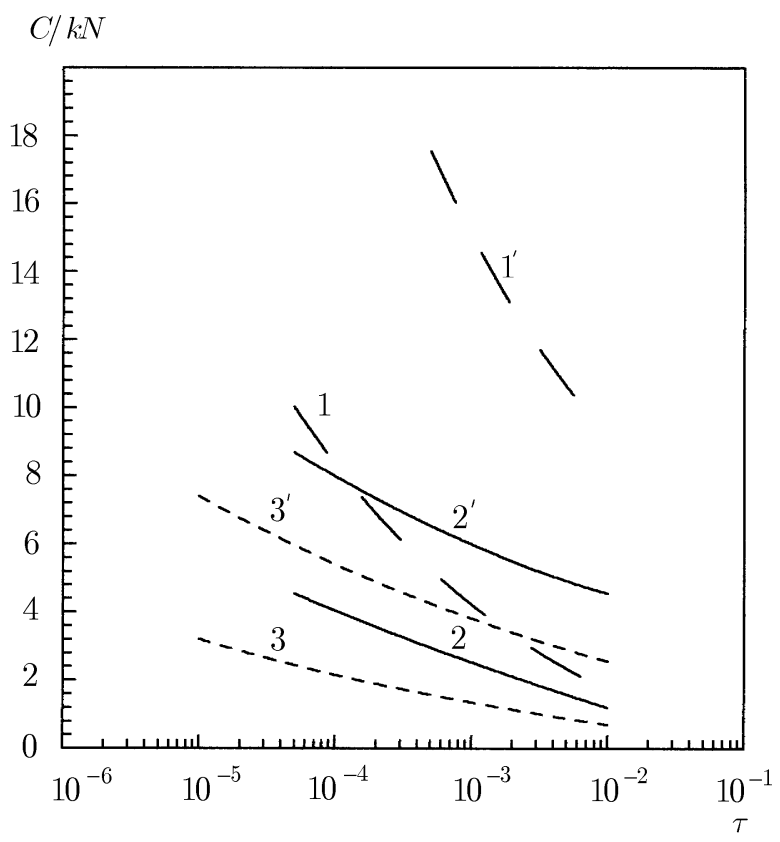

Рис. 10. Зависимость теплоемкости системы от $\tau=\left|T-T_{\mathrm{C}}\right| / T_{\mathrm{C}}$.

неаналитической зависимостью коэффициентов от температуры. Главной отличительной чертой данного метода расчета является раздельный учет вкладов в термодинамические функции от коротко- и длинноволновых мод колебаний плотности спинового момента $[11,86,87]$. Показано, что правильный температурньй ход энтропии и положительность теплоемкости обеспечиваются вкладом длинноволновых мод колебаний, играющих роль стабилизируюшего фактора. Коротковолновые моды, способствующие неустойчивости системы, ответственны за формирование значений критических показателей и перенормировку дисперсии распределения, описываюшего длинноволновые моды.

На основе первых принципов статистической физики построена целостная картина фазового перехода. Единым образом получены критические показатели, дано определение и рассчитана температура фазового перехода, найдены выражения для основных критических амплитуд и амплитуд конфлюэнтных поправок термодинамических характеристик $[11,86,87,89,95,101,102]$. Значения критических показателей, отношений критических амплитуд для теплоемкости и других характеристик системы, комбинаций этих амплитуд, температуры фазового перехода, вычисленные с использованием моделей $\rho^{4}$ и $\rho^{6}$, согласуются с данными теоретических и экспериментальных исследований. Так, например [95], критические показатели $\nu=0.637, \alpha=0.088$, $\beta=0.319, \gamma=1.275$ и показатель первой поправки к скейлингу $\Delta_{1}=0.525$ (модель $\left.\rho^{6}, s=s^{*}\right)$, а также отношения критических амплитуд теплоемкости $A^{+} / A^{-}=0.435$, восприимчивости $\Gamma^{+} / \Gamma^{-}=6.967$ и их комбинации $\mathcal{P}=\left[1-A^{+} / A^{-}\right] / \alpha=3.054, R_{\mathrm{c}}^{+}=$ $A^{+} \Gamma^{+} /\left[s_{0}^{3}\left(\langle\sigma\rangle^{(0)}\right)^{2}\right]=0.098$ (модель $\rho^{4}, s=s^{*}$ ) находятся в согласии со значениями $\nu=$ $0.630, \alpha=0.110, \beta=0.325, \gamma=1.241, \Delta_{1}=0.498, A^{+} / A^{-}=0.465, \Gamma^{+} / \Gamma^{-}=5.12$,

5 Теоретическая и математическая физика, т. 117, № 3, 1998 г. 
$\mathcal{P}=3.90, R_{\mathrm{c}}^{+}=0.052$, полученными в рамках теоретико-полевого подхода $[26,119,120]$, и со значениями $\nu=0.638, \alpha=0.125, \beta=0.312, \gamma=1.250, \Delta_{1}=0.50, A^{+} / A^{-}=0.51$, $\Gamma^{+} / \Gamma^{-}=5.07, R_{\mathrm{c}}^{+}=0.059$, рассчитанными с помощью высокотемпературного разложения [121-125]. Прямой аналитический способ расчета температуры фазового перехода развит в работах $[105,126]$. В указанных работах на основе четверной плотности меры изучается влияние на эту температуру коротковолновой части фурье-образа экспоненщиально убываюшего потенциала взаимодействия. Приводятся численные данные температуры фазового перехода для различных значений радиуса эффективного действия потенциала (в том числе и для значения, соответствующего учету взаимодействия ближайших соседей). Значение $J /\left(k T_{c}\right)=0.211$, полученное для взаимодействия ближайших соседей с постоянной $J$, согласуется с соответствующими значениями, рассчитанными другими методами (например, со значением $J /\left(k T_{\mathrm{c}}\right)=0.2216595$ [62, 63], вычисленным с использованием метода Монте-Карло).

Получение аналитических выражений для коэффициентов термодинамических характеристик как функций микроскопических параметров системы является преимушеством рассматриваемого метода. В процессе нахождения этих характеристик разработана методика расчета конфлюэнтных поправок: первой $[88,89,108,109]$ и второй $[88,89$, 110] в приближении четверной, а также первой $[101,102]$ в приближении шестерной плотностей мер. Слагаемые, определяющие первую конфлюэнтную поправку, вносят в термодинамические функции системы вблизи $T_{\text {c }}$ более сушественньй вклад по сравнению с вкладом от слагаемых, определяющих вторую поправку. В случае модели $\rho^{4}$ вычислен вклад от конфлюэнтных поправок в полную теплоемкость [89, 111], которая для различных микроскопических параметров системы представлена в работе [112]. Показано, что каждую из основных критических амплитуд и амплитуд конфлюэнтных поправок можно представить в виде произведения универсальной части, не зависящей от микроскопических параметров, и зависящего от этих параметров неуниверсального фактора. Наличие подобного неуниверсального множителя, входящего в состав амплитуд конфлюэнтных поправок теплоемкости, восприимчивости, коррелящионной длины при температурах выше и ниже критической, а также для параметра порядка, было установлено в методе $\epsilon$-разложения для $n$-компонентной системы [127]. Однако выражения, полученные в используемом нами методе, позволяют установить явную зависимость неуниверсального фактора от микроскопических параметров и рассчитать в зависимости от них термодинамические функции.

Результаты проведенных исследований и их сравнение с другими данными [95, 102, $128]$ свидетельствуют о том, что модель $\rho^{6}$ по сравнению с моделью $\rho^{4}$ обеспечивает лучшее количественное описание критических свойств трехмерной изинговской системы.

Рассмотрены специальные функции нового типа, появляюшиеся при построении теории фазового перехода с использованием шестерной плотности меры $[93,104,106]$. Эти функции, входяшие в РС для коэффищиентов мер последуюших блочных структур, представлены в виде быстросходяшихся рядов по степеням малых отклонений аргументов функций от их значений в фиксированной точке. Учитываются линейные отклонения и их квадраты $[95,113]$. Приводятся численные данные для коэффициентов таких представлений. 
На основе шестерной плотности меры предложен новый метод получения уравнения состояния трехмерной модели Изинга на простой кубической решетке с экспоненциально убывающим потенциалом взаимодействия [96, 128]. Это уравнение позволяет описать в критической области поведение параметра порядка как функцию температуры, внешнего поля и микроскопических параметров системы. Численное исследование температурной и полевой зависимости параметра порядка модели Изинга выполнено для случая потенциала взаимодействия ближайших соседей.

В рамках модели $\rho^{6}$ с учетом первой конфлюэнтной поправки исследована зависимость основных характеристик трехмерной изинговской системы от ее микроскопических параметров. Для разных значений радиуса действия экспоненциально убывающего потенциала взаимодействия (в том числе и для значений, соответствующих учету взаимодействия ближайших, первых и вторых, первых, вторых и третьих соседей) оценен размер критической области, рассчитаны основные критические амплитуды и амплитуды конфлюэнтной поправки теплоемкости и восприимчивости, приведены графики температурных зависимостей среднего спинового момента, энтропии, теплоемкости и восприимчивости системы. Прослежено поведение температуры фазового перехода, свободной энергии в точке фазового перехода, среднего спинового момента и теплоемкости с ростом отношения вышеуказанного радиуса к постоянной простой кубической решетки.

Работа выполнена при частичной финансовой поддержке Государственного фонда фундаментальных исследований Миннауки Украины (проект № 2.4/173).

\section{Список литературы}

[1] Р. Браут. Фазовые переходы. М.: Мир, 1967.

[2] М. Фищер. Природа критического состояния. М.: Мир, 1968.

[3] Ф. Дайсон, Э. Монтролл, М. Кач, М. Фишер. Устойчивость и фазовые переходы. М.: Мир, 1973.

[4] Г. Стенли. Фазовые переходы и критические явления. М.: Мир, 1973.

[5] К. Вильсон, Дж. Когут. Ренормализационная группа и $\epsilon$-разложение. М.: Мир, 1975.

[6] Phase Transitions and Critical Phenomena. V. 6. Eds C. Domb, M.S. Green. New York: Academic Press, 1976.

[7] Р. Балеску. Равновесная и неравновесная статистическая механика. Т. І. М.: Мир, 1978.

[8] ШI. Ма. Современная теория критических явлений. М.: Мир, 1980.

[9] Я. Г. Синай. Теория фазовых переходов: Строгие результаты. М.: Наука, 1980.

[10] Л.3. Паташинский, В. Л. Покровский. Флуктуационная теория фазовых переходов. М.: Наука, 1982.

[11] И. Р. Юхновский. Фазовые переходы второго рода. Метод коллективных переменных. Киев: Наукова думка, 1985.

[12] Phase Transitions and Critical Phenomena. V. 10. Eds C. Domb, J. L. Lebowitz. London: Academic Press, 1986.

[13] Н. Марч, М. Паринелло. Коллективные эффекты в твердых телах и жидкостях. М.: Мир, 1986.

[14] М. А. Анисимов. Критические явления в жидкостях и жидких кристаллах. М.: Наука, Гл. ред. физ.-мат. лит., 1987.

[15] Ю.М. Иванченко, А.А. Лисянский, А. Э. Филиппов. Флуктуационные эффекты в системах с конкурирующими взаимодействиями. Киев: Наукова думка, 1989.

[16] F. J. Wegner. Critical phenomena and scale invariance. In: Lect. Notes in Phys. V. 54. "Critical Phenomena" Sitges International School on Statistical Mechanics, June 1976, Sitges, Barcelona, 
Spain. Eds J. Grey and R. B. Jones. Berlin-Heidelberg-New York: Springer-Verlag, 1976. P. 1.

[17] Colin J. Thompson. Contemp. Phys. 1978. V. 19. № 3. P. 203.

[18] П. Флёри. УФН. 1982. Т. 138. № 1. С. 129.

[19] G. A. Baker, Jr. Quantitative Theory of Critical Phenomena. San Diego: Academic Press, Inc., 1990.

[20] J. J. Binney, N.J. Dowrick, A.J. Fisher, M.E. J. Newman. The Theory of Critical Phenomena. An Introduction to the Renormalization Group. Oxford: Clarendon Press, 1992.

[21] D. P. Landau. Physica A. 1994. V. 205. P. 41

[22] C. Domb. The Critical Point. A Historical Introduction to the Modern Theory of Critical Phenomena. London: Taylor \& Francis Ltd, 1996.

[23] Н. Н. Боголюбов, Д. В. Ширков. Введение в теорию квантованных полей. М.: Наука, 1984.

[24] D. J. Amit. Field Theory, the Renormalization Group, and Critical Phenomena. Singapore: World Scientific, 1984.

[25] G. Parisi. J. Stat. Phys. 1980. V. 23. № 1. P. 49.

[26] J. C. Le Guillou, J. Zinn-Justin. Phys. Rev. B. 1980. V. 21. № 9. P. 3976.

[27] J. C. Le Guillou, J. Zinn-Justin. J. Phys. Lett. (FR). 1985. V. 46. № 4. P. 137.

[28] Р. Бәкстер. Точно решаемые модели в статистической механике. М.: Мир, 1985.

[29] D. S. Gaunt. J. Phys. A. 1986. V. 19. № 3. P. L149.

[30] Gregory C. Psaltakis. Phys. Rev. B. 1989. V. 39. № 4. P. 2834.

[31] И. А. Вакарчук, Ю. К. Рудавский. ТМФ. 1981. Т. 49. № 2. С. 234.

[32] И. А. Вакариук, Ю. К. Рудавский, И. Р. Юхновский. ТМФ. 1982. Т. 50. № 2. С. 313.

[33] И. А. Вакарчук, Ю. К. Рудавский. ТМФ. 1982. Т. 51. № 1. С. 102.

[34] W. Reich, H. Cofta. Phys. Stat. Sol. (b). 1986. V. 134. № 1. P. K31.

[35] W. Reich. Phys. Stat. Sol. (b). 1988. V. 149. № 2. P. K163.

[36] K. G. Chakraborty, J. W. Tucker. Physica A. 1986. V. 137. № 1-2. P. 111.

[37] J. R. Faleiro Ferreira. Phys. Stat. Sol. (b). 1988. V. 148. № 2. P. 709.

[38] С. В. Тябликов. Методы квантовой теории магнетизма. М.: Наука, 1975.

[39] В. И. Горбатюк. Функция Грина в модели Изинга. В сб.: Материаловед. узкощелев. и слоист. полупровод. Ред. К. Д. Товстюк и др. Киев: Наукова думка, 1989.

[40] И. М. Дубровский. ФТТ (Ленинград). 1990. Т. 33. № 12. С. 3629.

[41] PL. Cavalotti, M. Alberti, B. Bozzini, A. Iudica, L. Nobili, P. M. Ossi. J. Magn. Magn. Mater. 1992. V. 104-107. № 2. P. 905.

[42] J. Mattson, C. Djurberg, P. Nordblad. J. Magn. Magn. Mater. 1994. V. 136. P. L23.

[43] Н. П. Маломуж, А. И. Фисенко. УФЖ. 1989. Т. 34. № 7. С. 1043.

[44] N. P. Malomuzh, B. A. Veytsman. Physica A. 1990. V. 168. № 2. P. 833.

[45] Б. А. Вейцман, Н. П. Маломуж. ЖХФХ. 1989. Т. LXIII. № 11. С. 3068.

[46] С. В. Павлов. Вестн. МГУ. Сер. 3. 1990. Т. 31. № 1. С. 70.

[47] L. P. Kadanoff. Physica A. 1990. V. 163. № 1. P. 1.

[48] G. Lei. J. Comput. Phys. 1991. V. 92. № 1. P. 106.

[49] H. Mano, K. Nakao. J. Phys. Soc. Japan. 1991. V. 60. № 2. P. 548.

[50] Y. Kinosita, N. Kawashima, M. Suzuki. J. Phys. Soc. Japan. 1992. V. 61. № 11. P. 3887.

[51] C. J. Thompson. Progr. Theor. Phys. 1992. V. 87. № 3. P. 535.

[52] R. Hilfer. Mod. Phys. Lett. B. 1992. V. 6. № 13. P. 773.

[53] A. J. Liu, M. E. Fisher. Physica A. 1989. V. 156. № 1. P. 35.

[54] A. J. Liu, M. E. Fisher. J. Stat. Phys. 1990. V. 58. № 3-4. P. 431.

[55] J. Lee, J. M. Kosterlitz. Phys. Rev. Lett. 1990. V. 65. № 2. P. 137.

[56] M. A. Novotny. J. Appl. Phys. 1990. V. 67. № 9. Pt 2B. P. 5448.

[57] H. W. J. Blöte, A. Compagner, J. H. Croockewit, Y. T. J. C. Fonk, J. R. Heringa, A. Hoogland, T.S. Smit, A. L. Van Willigen. Physica A. 1989. V. 161. № 1. P. 1.

[58] Lai Pik-Yin, K. K. Mon. Phys. Rev. B. 1989. V. 10. № 16. P. 11120.

[59] A. G. Schlijper, A. R. D. Van Bergen, B. Smit. Phys. Rev. A. 1990. V. 41. № 2. P. 1175.

[60] F. Livet. Europhys. Lett. 1991. V. 16. № 2. P. 139.

[61] C.F. Baillie. Comput. Phys. Commun. 1991. V. 65. № 1-3. P. 17.

[62] A. M. Ferrenberg, D. P. Landau. Phys. Rev. B. 1991. V. 44. № 10. P. 5081. 
[63] D. P. Landau, A. M. Ferrenberg. J. Kor. Phys. Soc. 1993. V. 26. P. S371.

[64] M. Caselle, M. Hasenbusch. J. Phys. A. 1997. V. 30. P. 4963.

[65] T. Horiguchi, O. Nagai, S. Miyashita. J. Phys. Soc. Japan. 1992. V. 61. № 1. P. 308.

[66] G. Bhanot, M. Creutz, U. Glässner, K. Schilling. Phys. Rev. B. 1994. V. 49. № 18. P. 12909.

[67] И. Р. Юхновский. ДАН СССР. 1977. Т. 232. № 2. С. 312.

[68] И. Р. Юхновский. ТМФ. 1978. Т. 36. № 3. С. 373.

[69] I. R. Yukhnovs'kii. Riv. Nuovo Cimento. 1989. V. 12. № 1. P. 1.

[70] Д. Бом. Общая теория коллективных переменных. М.: Мир, 1964.

[71] Д. Н. Зубарев. ДАН СССР. 1954. Т. 95. № 4. С. 757.

[72] Н. Н. Боголюбов, Д. Н. Зубарев. ЖЭТФ. 1955. Т. 28. № 2. С. 129.

[73] М. В. Ваврух. ТМФ. 1978. Т. 36. № 3. С. 400.

[74] И. Р. Юхновский, М. Ф. Головко. Статистическая теория классических равновесных систем. Киев: Наукова думка, 1980.

[75] И. А. Вакарчук, Г. В. Понедилок, Ю. К. Рудавский. ТМФ. 1984. Т. 58. № 3. С. 445.

[76] Л. Ф. Блажиевский. УФЖ. 1986. Т. 31. № 2. С. 297.

[77] М. В. Ваврух. ТМФ. 1982. Т. 50. № 3. С. 438.

[78] И. Р. Юхновский, З. А. Гурский. Квантово-статистическая теория неупорядоченных систем. Киев: Наукова думка, 1991.

[79] I. R. Yukhnovskii, N. A. Korynevskii. Phys. Stat. Sol. (b). 1991. V. 163. P. 355.

[80] I. R. Yukhnovskii, I. M. Idzyk, V. O. Kolomiets. J. Stat. Phys. 1995. V. 80. № 1-2. P. 405.

[81] I. R. Yukhnovskii, O. V. Patsahan. J. Stat. Phys. 1995. V. 81. № 3-4. P. 647.

[82] И.Р. Юхновский, Ю. К. Рудавский, Ю. В. Головач. Термодинамика необратимых процессов. М.: Наука, 1987.

[83] М. П. Козловский, З. Е. Усатенко. УФЖ. 1997. Т. 42. № 3. С. 368.

[84] Ya. M. Ilnytskyi, M.P. Kozlovskii, I. R. Yukhnovskii. Int. J. Mod. Phys. B. 1997. V. 11. № 8. P. 1009 .

[85] I. R. Yukhnovskii. Phase Transitions of the Second Order. Collective Variables Method. Singapore: World Scientific, 1987.

[86] M.P. Kozlovsky, I. V. Pylyuk. Free energy and other thermodynamical functions above the second-order phase transition point. Preprint ITP-85-23E. Kiev: ITP Acad. Sci. Ukr. SSR, 1985 .

[87] I. R. Yukhnovskii, M.P. Kozlovskii, I. V. Pylyuk. Z. Naturforsch. 1991. V. 46a. P. 1.

[88] М. П. Козловский, И. В. Пылюк, И. Р. Юхновский. ТМФ. 1991. Т. 87. № 2. С. 293.

[89] М. П. Козловский, И. В. Пылюк, И. Р. Юхновский. ТМФ. 1991. Т. 87. № 3. С. 434.

[90] И. В. Пьлюк, М. П. Козловский. Исследование модели Изинга с использованием негауссовых базисных мер. Препринт ИТФ-87-31Р. Киев: ИТФ АН УССР, 1987.

[91] И. В. Пьлюк. Критическое поведение трехмерной однокомпонентной спиновой системы в методе коллективных переменных при усложнении базисной меры. Препринт ИТ $\Phi-88-107 \mathrm{P}$. Киев: ИТФ АН УССР, 1988.

[92] М. П. Козловский, И. В. Пьлюк. Расчет критического показателя корреляционной длины трехмерной модели Изинга с использованием негауссовых базисних мер. В сб.: Тр. Всесоюзной конф. "Современные проблемы статистической физики". Т. 2. Киев: Наукова думка, 1989. C. 50.

[93] М. П. Козловский. ТМФ. 1989. Т. 78. № 3. С. 422.

[94] М. П. Козловський, И. В. Пьлюк.УФЖ. 1990. Т. 35. № 1. С. 146.

[95] М. П. Козловский, И. В. Пылюк. Термодинамика трехмерного изинговского ферромагнетика в окрестности точки фазового перехода в рамках модели $\rho^{6}$. Сравнение с моделью $\rho^{4}$. Препринт ИТФ-90-81Р. Киев: ИТФ АН УССР, 1990.

[96] В. В. Духовый, М. П. Козловский, И. В. Пълюк. ТМФ. 1996. Т. 107. № 2. С. 288.

[97] R. Guida, J. Zinn-Justin. Nucl. Phys. B. [FS]. 1997. V. 489. P. 626.

[98] M. M. Tsypin. Phys. Rev. Lett. 1994. V. 73. № 15. P. 2015.

[99] A. I. Sokolov, V. A. Ul’kov, E. V. Orlov. J. Phys. Stud. (Lviv). 1997. V. 1. № 3. P. 362.

[100] A. I. Sokolov, E. V. Orlov, V. A. Ul'kov. Phys. Lett. A. 1997. V. 227. P. 255. 
[101] И. В. Пылюк, М. П. Козловский. Термодинамические характеристики трехмерной изинговской системы в приближении модели $\rho^{6}$ с учетом конфлуентной поправки. І. Высокотемпературная область. Препринт ICMP-97-06U. Львов: ИФКС НАН Украины, 1997.

[102] И. В. Пылюк, М. П. Козловский. Термодинамические характеристики трехмерной изинговской системы в приближении модели $\rho^{6}$ с учетом конфолентной поправки. II. Низкотемпературная область. Препринт ICMР-97-07U. Львов: ИФКС НАН Украины, 1997.

[103] М. П. Козловский. Критические свойства модели Изинга. Модель $\rho^{6}$. Общие рекуррентные соотношения. Препринт ИТФ-82-104Р. Киев: ИТФ АН УССР, 1982.

[104] М. П. Козловский. Решения уравнений ренормгруппы для системы изинговских спинов в модели $\rho^{6}$. Препринт ИТФ-84-35Р. Киев: ИТФ АН УССР, 1984.

[105] M. P. Kozlovskii, I. V. Pylyuk, Z. E. Usatenko. Phys. Stat. Sol. (b). 1996. V. 197. P. 465.

[106] М. П. Козловский, И. В. Пылюк. Расчет термодинамических функций вблизи точки фазового перехода в приближении шестерной базисной меры. Препринт ИТФ-87-9Р. Киев: ИТФ AH УCCP, 1987.

[107] М. П. Козловский, Я. Н. Ильницкий, И. В. Пьлюк. Свободная энергия и другие термодинамические функции трехмерной модели Изинга ниже точки фазового перехода. Препринт ИТФ-85-107Р. Киев: ИТФ АН УССР, 1985.

[108] М. П. Козловский, И. В. Пьлюк. Расчет поправок к скейлингу в выражениях для термодинамических функций модели Изинга. Препринт ИТФ-89-24Р. Киев: ИТФ АН УССР, 1989.

[109] М. П. Козловский, И. В. Пьлюк. Сравнение выражений для термодинамических функций модели Изинга при температурах выше и ниже критической в трехмерном пространстве. Препринт ИТФ-89-42Р. Киев: ИТФ АН УССР, 1989.

[110] И. В. Пьлюк. Термодинамические функции трехмерного изинговского ферромагнетика в окрестности точки фазового перехода с учетом первой и второй конфлуентных поправок. Препринт ИТФ-90-12Р. Киев: ИТФ АН УССР, 1990.

[111] И. В. Пьлююк, М. П. Козловский. Изв. АН СССР. Сер. физич. 1991. Т. 55. № 3. С. 597.

[112] M. P. Kozlovskii, I. V. Pylyuk. Phys. Stat. Sol. (b). 1994. V. 183. P. 243.

[113] И. В. Пылюю.. УФЖК. 1996. Т. 41. № 9. С. 885.

[114] C. Bagnuls, C. Bervillier. Phys. Rev. B. 1981. V. 24. № 3. P. 1226.

[115] J. F. Nicoll, P. C. Albright. Phys. Rev. B. 1986. V. 34. № 3. P. 1991.

[116] М. П. Козловский, И. В. Пьлюк, В.А. Коломиец. Численное исследование статистической суммы трехмерной модели Изинга на основе шестерного базисного распределения. Препринт ИТФ-84-177Р. Киев: ИТФ АН УССР, 1984.

[117] И. Р. Юхновский, М. П. Козловский, И. В. Пьлюк. Учет поправки на усреднение потенциала в методе поэтапного вычисления статсуммы однокомпонентной спиновой системы вблизи $T_{\mathrm{c}}$. Препринт ИТФ-88-105Р. Киев: ИТФ АН УССР, 1988.

[118] И. Р. Юхновский, М. П. Козловский. Корреляционная функция трехмерной модели Изинга вблизи точки фазового перехода. Препринт ИТФ-89-69Р. Киев: ИТФ АН УССР, 1989.

[119] C. Bagnuls, C. Bervillier, E. Boccara. Phys. Lett. A. 1984. V. 103. № 9. P. 411.

[120] M. Barmatz, P. C. Hohenberg, A. Kornblit. Phys. Rev. B. 1975. V. 12. № 5. P. 1947.

[121] D. S. Gaunt, M. F. Sykes. J. Phys. A. 1973. V. 6. № 10. P. 1517.

[122] D. M. Saul, M. Wortis, D. Jasnov. Phys. Rev. B. 1975. V. 11. № 7. P. 2571.

[123] W. J. Camp, J. P. Van Dyke. Phys. Rev. B. 1975. V. 11. № 7. P. 2579.

[124] A. Aharony, P. C. Hohenberg. Phys. Rev. B. 1976. V. 13. № 7. P. 3081.

[125] W. J. Camp, D. M. Saul, J.P. Van Dyke, M. Wortis. Phys. Rev. B. 1976. V. 14. № 9. P. 3990.

[126] М. П. Козловский, И. В. Пълюю, З Е. Усатенко. УФЖ. 1995. Т. 40. № 10. С. 1141.

[127] J. F. Nicoll, P. C. Albright. Phys. Rev. B. 1985. V. 31. № 7. P. 4576.

[128] M. P. Kozlovskii, I. V. Pylyuk, V. V. Dukhovii. J. Magn. Magn. Mater. 1997. V. 169. P. 335. 